\title{
OPEN Effects of different salt sources and salinity levels on emergence and seedling growth of faba bean genotypes
}

\author{
Nurlykhan Bimurzayev ${ }^{1}$, Hatice Sari ${ }^{2}$, Ahmet Kurunc ${ }^{1}{ }^{1 凶}$, Kıvanc Hayri Doganay ${ }^{1}$ \& \\ Mulat Asmamaw ${ }^{1}$
}

Suitability of poor quality water for irrigation depends on salinity level and solute concentration in the water and selected crop. Salt stress is a major potential constraint for faba bean. The present study aimed to investigate the effects of different $\mathrm{Cl}$ - and $\mathrm{SO}_{4}$-containing salt sources in irrigation water with different salinity levels on emergence, early seedling growth and photosynthetic capacity of six faba bean genotypes. The negative effect order of salinity level was high $(3 \mathrm{dS} / \mathrm{m})>$ medium $(2 \mathrm{dS} / \mathrm{m})>\operatorname{low}(1 \mathrm{dS} / \mathrm{m})>$ control $(0.05 \mathrm{dS} / \mathrm{m})$ for all investigated parameters except dry root weight. The negative effects of $\mathrm{Cl}$-containing salt sources were higher than that of $\mathrm{SO}_{4}$-containing salt sources. The worst and the best performing genotypes were determined as III-28 and III-29 on emergence percentage at 10th DAS, I-29 and III-1 on mean emergence time, III-22 and III-1 on shoot height, III-1 and I-29 on fresh biomass weight, III-22 and III-28 on fresh shoot weight, III-29 and I-29 on fresh root weight, respectively. This study showed that faba bean genotypes have different behaviors in terms of response to the increasing salinity levels artificially makeup by using different salt sources indicating that salt response of faba bean is genotype-specific.

Faba bean (Vicia faba L.) also referred to as broad bean, horse bean or field bean, is a good source of protein, energy and fiber and the crop is widely grown for food and feed ${ }^{1}$. It is used as a source of protein in human diets, as fodder and a forage crop for animals, and for its excellent ability to fix atmospheric nitrogen. Seeds of faba bean are consumed in large quantities in developing countries such as China, Turkey, Egypt, Ethiopia, and Central America ${ }^{2}$. In addition to a good alternative to animal proteins, it is also an attractive product because of its low cost, long storage life, and easy transportation ${ }^{3}$. In terms of world production, faba bean ranked sixth with 4.5 Mt from 2.5 Mha after common bean (Phaseolus vulgaris L.), pea (Pisum sativum L.), chickpea (Cicer arietinum L.), cowpea (Vigna unguiculata L. Walp.), and lentil (Lens culinaris Medik.) ${ }^{4}$. Faba bean is the fourth most widely grown pulse crop in Turkey ${ }^{5}$. In Turkey, the cultivated area of faba bean seeds for food and feed are 2312 and 2020 ha in 2019 with the average yields of 2.37 and 3.40 tons per hectare and with total productions of 5484 and 6862 tons, respectively ${ }^{6}$.

All agricultural soils and irrigation water contain mineral salts. The amount and kind of salt present depends on the makeup of both the soil and irrigation water ${ }^{7}$. The ionic composition of irrigation water is as important as the level of water salinity. Each type of salt in the soil and irrigation water may exert different effects on plant growth ${ }^{8}$. Particularly in the semi-arid regions of the world, such as the Mediterranean Sea, the supply of sufficient quality water for irrigation is an important problem. This situation is leading to the use of groundwater containing excessive amounts of soluble salts with chlorine compounds in the coastal areas ${ }^{9}$. In contrast, the water sources in the inner parts contains sulfide compounds. It was reported that electrical conductivity values of groundwater used for irrigation purposes in greenhouses in Antalya, ranged from 0.85 to $4.1 \mathrm{dS} / \mathrm{m}$ in November (winter) to $0.83-4.4 \mathrm{dS} / \mathrm{m}$ in June (summer) ${ }^{10}$.

Use of poor quality or saline water for irrigation is a subject of increasing interest for sustaining crop productivity. Although salinity affects the crops at all growth stages of development, plant sensitivity to salinity for most crops varies from one stage to the next. Most of the salinity effect on crops were obtained from salinity treatments imposed after seedlings were established in non-saline plots and do not necessarily apply to germination,

${ }^{1}$ Department of Agricultural Structures and Irrigation, Faculty of Agriculture, Akdeniz University, Campus, 07058 Antalya, Turkey. ${ }^{2}$ Department of Field Crops, Faculty of Agriculture, Akdeniz University, Campus, 07058 Antalya, Turkey. ${ }^{\bowtie}$ email: akurunc@akdeniz.edu.tr 


\begin{tabular}{|c|c|c|c|c|c|c|c|}
\hline \multirow[b]{3}{*}{ Source of variation } & \multicolumn{7}{|c|}{ Mean square } \\
\hline & \multicolumn{3}{|c|}{$\begin{array}{l}\text { Emergence percentage @ } \\
\text { DAS }\end{array}$} & \multirow{2}{*}{\begin{tabular}{|l} 
Mean Emergence Time \\
(day)
\end{tabular}} & \multirow{2}{*}{\begin{tabular}{|l|} 
Shoot height \\
(cm)
\end{tabular}} & \multicolumn{2}{|c|}{$\begin{array}{l}\text { Biomass weight } \\
\text { (g) }\end{array}$} \\
\hline & 10th & 17th & 24th & & & Fresh & Dry \\
\hline Genotype (G) & $<0.001$ & $<0.001$ & $<0.001$ & $<0.001$ & $<0.001$ & $<0.001$ & $<0.001$ \\
\hline Salt source (S) & $<0.001$ & 0.152 & 0.602 & $<0.001$ & $<0.001$ & $<0.001$ & $<0.001$ \\
\hline Salinity level (L) & $<0.001$ & $<0.001$ & 0.002 & $<0.001$ & $<0.001$ & $<0.001$ & $<0.001$ \\
\hline $\mathrm{G} \times \mathrm{S}$ & 0.024 & 0.112 & 0.041 & 0.208 & $<0.001$ & $<0.001$ & 0.002 \\
\hline $\mathrm{G} \times \mathrm{L}$ & $<0.001$ & $<0.001$ & 0.002 & $<0.001$ & $<0.001$ & $<0.001$ & $<0.001$ \\
\hline $\mathrm{S} \times \mathrm{L}$ & $<0.001$ & 0.030 & 0.046 & $<0.001$ & $<0.001$ & $<0.001$ & $<0.001$ \\
\hline $\mathrm{G} \times \mathrm{S} \times \mathrm{L}$ & 0.211 & 0.597 & 0.373 & 0.677 & 0.189 & 0.101 & 0.802 \\
\hline \multirow[b]{3}{*}{ Source of variation } & \multicolumn{7}{|c|}{ Mean square } \\
\hline & \multicolumn{2}{|c|}{ Shoot weight (g) } & \multicolumn{2}{|c|}{ Root weight (g) } & \multirow{2}{*}{\multicolumn{3}{|c|}{$F_{v} / F_{m}$}} \\
\hline & Fresh & Dry & Fresh & Dry & & & \\
\hline Genotype (G) & $<0.001$ & $<0.001$ & $<0.001$ & $<0.001$ & $<0.001$ & & \\
\hline Salt source (S) & $<0.001$ & $<0.001$ & $<0.001$ & $<0.001$ & $<0.001$ & & \\
\hline Salinity level (L) & $<0.001$ & $<0.001$ & $<0.001$ & $<0.001$ & $<0.001$ & & \\
\hline $\mathrm{G} \times \mathrm{S}$ & 0.009 & 0.349 & $<0.001$ & $<0.001$ & 0.081 & & \\
\hline $\mathrm{G} \times \mathrm{L}$ & $<0.001$ & $<0.001$ & $<0.001$ & $<0.001$ & 0.616 & & \\
\hline $\mathrm{S} \times \mathrm{L}$ & $<0.001$ & $<0.001$ & $<0.001$ & $<0.001$ & $<0.001$ & & \\
\hline $\mathrm{G} \times \mathrm{S} \times \mathrm{L}$ & 0.341 & 0.746 & 0.005 & 0.225 & 0.332 & & \\
\hline
\end{tabular}

Table 1. $P$ values (significance) from analysis of variance for the effects of faba bean genotype, salt source and salinity level on investigated parameters.

emergence and early seedling stages ${ }^{11,12}$. Salinity is also known to impair seed germination, emergence and early seedling growth. In addition, in many studies on salt tolerance, it is not been possible to determine the detrimental effects on growth that is associated with whether the level of salinity or the kind of salt present in the water or soil solution. Therefore, the exploitation of seed performance under salt and salinity effect on emergence and early seedling growth stage will be helpful for improving the choice of genotype suited to perform best under known conditions of salinity ${ }^{13}$.

Faba bean has a bright future as a protein crop that provides additional crop rotation benefits for many areas of the world. However, salinity is an ever-present major constraint and a major threat to these kind of legume crops, particularly in areas with irrigated agriculture ${ }^{14}$. This plant is known to be moderately sensitive to salinity and should, therefore, be cultivated on soils with little or no saline content ${ }^{11}$. The harmful effects have been reported for faba bean genotypes under saline conditions composed of $\mathrm{NaCl}^{15-17}$. However, no information is available regarding the comparison of $\mathrm{CaCl}_{2}, \mathrm{MgCl}_{2}, \mathrm{CaSO}_{4}, \mathrm{MgSO}_{4}$ and $\mathrm{Na}_{2} \mathrm{SO}_{4}$ salts and salinity levels. Ayers and Westcot ${ }^{12}$ claim that the suitability of water for irrigation should be determined by the amount and kind of salt present. Also, emergence percentage and early growth of crops under a particular level of salt vary considerably among genotypes ${ }^{18}$. Therefore, the present study aimed to evaluate the effects of different salt sources and irrigation water salinity levels on the emergence and early growth parameters of six faba bean genotypes.

\section{Results}

The results of ANOVA for faba bean genotypes (G), salt sources (S) and salinity levels (L) as the main factors, and their interactions including $\mathrm{G} \times \mathrm{S}, \mathrm{G} \times \mathrm{L}, \mathrm{S} \times \mathrm{L}$ and $\mathrm{G} \times \mathrm{S} \times \mathrm{L}$ were given in Table 1 . According to these statistical analyses, faba bean genotypes and salinity level as the main factors and $\mathrm{S} \times \mathrm{L}$ interaction had significant effects on all investigated parameters including emergence percentage at 10th, 17th $(P<0.05$ for S $\times \mathrm{L})$ and 24 th $(P<0.05$ for $\mathrm{S} \times \mathrm{L})$ DAS, mean emergence time; shoot height; fresh and dry biomass, shoot and root weights; and $\mathrm{F}_{\mathrm{v}} / \mathrm{F}_{\mathrm{m}}$ ratio. However, emergence percentage at 17 th and 24 th DAS for salt sources as the main effect; emergence percentage at 17 th DAS, mean emergence time, dry shoot weight and $\mathrm{F}_{\mathrm{v}} / \mathrm{F}_{\mathrm{m}}$ ratio for $\mathrm{G} \times \mathrm{S}$ interaction; and only $\mathrm{F}_{\mathrm{v}} / \mathrm{F}_{\mathrm{m}}$ ratio for $\mathrm{G} \times \mathrm{L}$ interaction were not statistically significant (Table 1). Since a three-way ANOVA did not show significant differences for all parameters (except fresh root weight), the results were interpreted by following two-way interaction of $S \times \mathrm{L}$ for each faba bean genotype and also by plotting $\mathrm{S} \times \mathrm{G}$ and $\mathrm{L} \times \mathrm{G}$ interactions by ignoring salinity level and salt sources, respectively ${ }^{19}$. Therefore, the performances of each faba bean genotype under $\mathrm{S} \times \mathrm{L}$ interaction, and individual effect of salinity levels averaged over salt sources and salt sources averaged over salinity level for all faba bean genotypes were evaluated.

Performance of I-29 faba bean genotype. Considering $\mathrm{S} \times \mathrm{L}$ interaction, $\mathrm{F}_{\mathrm{v}} / \mathrm{F}_{\mathrm{m}}$ ratios showed a significant difference for I-29 genotype, whereas emergence percentage at 10th (ranged between 0.0 and 26.7\%), and at 24th (ranged between 63.3 and 100.0\%) DAS, mean emergence time (ranged between 11.47 and 15.77 days), shoot height (ranged between 4.51 and $9.23 \mathrm{~cm}$ ), fresh biomass weight (ranged between 6.73 and $12.40 \mathrm{~g}$ ), dry biomass weight (ranged between 1.03 and $1.51 \mathrm{~g}$ ), fresh shoot weight (ranged between 2.35 and $5.40 \mathrm{~g}$ ), dry shoot weight (ranged between 0.31 and $0.71 \mathrm{~g}$ ), fresh root weight (ranged between 4.38 and $7.56 \mathrm{~g}$ ) and dry root 


\begin{tabular}{|c|c|c|c|c|c|c|c|}
\hline \multirow[b]{2}{*}{ Parameter } & \multirow[b]{2}{*}{ Salinity level (dS/m) } & \multicolumn{6}{|c|}{ Salt source } \\
\hline & & $\mathrm{CaCl}_{2}$ & $\mathrm{MgCl}_{2}$ & $\mathrm{NaCl}$ & $\mathrm{CaSO}_{4}$ & $\mathrm{MgSO}_{4}$ & $\mathrm{Na}_{2} \mathrm{SO}_{4}$ \\
\hline \multirow{4}{*}{ Emergence percentage at 10th DAS } & 0.0 & $20.00^{x} a^{y}$ & $20.00 \mathrm{a}$ & $20.00 \mathrm{a}$ & $20.00 \mathrm{a}$ & $20.00 \mathrm{a}$ & $20.00 \mathrm{a}$ \\
\hline & 1.0 & $0.00 \mathrm{a}$ & $23.33 a$ & $26.67 \mathrm{a}$ & $20.00 \mathrm{a}$ & $26.67 \mathrm{a}$ & $10.00 \mathrm{a}$ \\
\hline & 2.0 & $10.00 \mathrm{a}$ & $3.33 \mathrm{a}$ & $6.66 \mathrm{a}$ & $20.00 \mathrm{a}$ & $30.00 \mathrm{a}$ & $16.67 \mathrm{a}$ \\
\hline & 3.0 & $0.00 \mathrm{a}$ & $3.33 \mathrm{a}$ & $3.33 \mathrm{a}$ & $20.00 \mathrm{a}$ & $16.67 \mathrm{a}$ & $26.67 \mathrm{a}$ \\
\hline \multirow{4}{*}{ Emergence percentage at 24th DAS } & 0.0 & $93.33 \mathrm{a}$ & $93.33 a$ & $93.33 \mathrm{a}$ & $93.33 a$ & $93.33 a$ & $93.33 a$ \\
\hline & 1.0 & $93.33 \mathrm{a}$ & $76.67 \mathrm{a}$ & $90.00 \mathrm{a}$ & 93.33a & $90.00 \mathrm{a}$ & $86.67 \mathrm{a}$ \\
\hline & 2.0 & $90.00 \mathrm{a}$ & $76.67 \mathrm{a}$ & $80.00 \mathrm{a}$ & $83.33 a$ & $100.00 \mathrm{a}$ & $86.67 \mathrm{a}$ \\
\hline & 3.0 & $63.33 \mathrm{a}$ & $70.00 \mathrm{a}$ & $73.33 \mathrm{a}$ & $76.67 \mathrm{a}$ & $90.00 \mathrm{a}$ & $83.33 \mathrm{a}$ \\
\hline \multirow{4}{*}{ Mean emergence time (day) } & 0.0 & $12.00 \mathrm{a}$ & $12.00 \mathrm{a}$ & $12.00 \mathrm{a}$ & $12.00 \mathrm{a}$ & $12.00 \mathrm{a}$ & $12.00 \mathrm{a}$ \\
\hline & 1.0 & $11.56 \mathrm{a}$ & $11.98 \mathrm{a}$ & $12.22 \mathrm{a}$ & $11.53 \mathrm{a}$ & $11.47 \mathrm{a}$ & $12.92 \mathrm{a}$ \\
\hline & 2.0 & $12.98 \mathrm{a}$ & $13.12 \mathrm{a}$ & $13.84 \mathrm{a}$ & $12.17 \mathrm{a}$ & $11.70 \mathrm{a}$ & $11.89 \mathrm{a}$ \\
\hline & 3.0 & $15.77 \mathrm{a}$ & $15.28 \mathrm{a}$ & $13.08 \mathrm{a}$ & $13.42 \mathrm{a}$ & $12.81 \mathrm{a}$ & $12.60 \mathrm{a}$ \\
\hline \multirow{4}{*}{ Shoot height $(\mathrm{cm})$} & 0.0 & $9.23 \mathrm{a}$ & $9.23 \mathrm{a}$ & $9.23 \mathrm{a}$ & $9.23 \mathrm{a}$ & $9.23 \mathrm{a}$ & $9.23 \mathrm{a}$ \\
\hline & 1.0 & $8.61 \mathrm{a}$ & $7.08 \mathrm{a}$ & $8.12 \mathrm{a}$ & $6.65 \mathrm{a}$ & $8.39 a$ & $6.60 \mathrm{a}$ \\
\hline & 2.0 & 6.46a & $5.05 a$ & $5.52 \mathrm{a}$ & $7.45 \mathrm{a}$ & $6.97 \mathrm{a}$ & $7.29 \mathrm{a}$ \\
\hline & 3.0 & $4.55 \mathrm{a}$ & $4.81 \mathrm{a}$ & $4.51 \mathrm{a}$ & $6.58 \mathrm{a}$ & $5.67 \mathrm{a}$ & $4.51 \mathrm{a}$ \\
\hline \multirow{4}{*}{ Fresh biomass weight (g) } & 0.0 & $11.17 \mathrm{a}$ & $11.17 \mathrm{a}$ & $11.17 \mathrm{a}$ & $11.17 \mathrm{a}$ & $11.17 \mathrm{a}$ & $11.17 \mathrm{a}$ \\
\hline & 1.0 & $12.40 \mathrm{a}$ & $10.20 \mathrm{a}$ & $12.02 \mathrm{a}$ & $10.13 a$ & $11.26 \mathrm{a}$ & $10.22 \mathrm{a}$ \\
\hline & 2.0 & $9.35 \mathrm{a}$ & 7.95a & $8.47 \mathrm{a}$ & $11.18 \mathrm{a}$ & 9.56a & $10.95 a$ \\
\hline & 3.0 & $7.75 a$ & $6.73 a$ & $8.22 \mathrm{a}$ & $11.79 a$ & 7.41a & $7.12 \mathrm{a}$ \\
\hline \multirow{4}{*}{ Dry biomass weight (g) } & 0.0 & $1.39 \mathrm{a}$ & $1.39 \mathrm{a}$ & $1.39 \mathrm{a}$ & $1.39 \mathrm{a}$ & $1.39 \mathrm{a}$ & $1.39 \mathrm{a}$ \\
\hline & 1.0 & $1.39 \mathrm{a}$ & $1.15 \mathrm{a}$ & $1.40 \mathrm{a}$ & $1.17 \mathrm{a}$ & $1.41 \mathrm{a}$ & $1.34 \mathrm{a}$ \\
\hline & 2.0 & $1.17 \mathrm{a}$ & $1.21 \mathrm{a}$ & $1.03 \mathrm{a}$ & $1.19 \mathrm{a}$ & $1.48 \mathrm{a}$ & $1.51 \mathrm{a}$ \\
\hline & 3.0 & $1.03 \mathrm{a}$ & $1.20 \mathrm{a}$ & $1.12 \mathrm{a}$ & $1.42 \mathrm{a}$ & $1.37 \mathrm{a}$ & $1.13 \mathrm{a}$ \\
\hline \multirow{4}{*}{ Fresh shoot weight (g) } & 0.0 & $4.60 \mathrm{a}$ & $4.60 \mathrm{a}$ & $4.60 \mathrm{a}$ & $4.60 \mathrm{a}$ & $4.60 \mathrm{a}$ & $4.60 \mathrm{a}$ \\
\hline & 1.0 & $5.40 \mathrm{a}$ & $4.41 \mathrm{a}$ & $4.77 \mathrm{a}$ & $3.78 \mathrm{a}$ & $4.74 \mathrm{a}$ & $4.14 \mathrm{a}$ \\
\hline & 2.0 & $4.20 \mathrm{a}$ & $3.22 \mathrm{a}$ & $3.53 a$ & $4.52 \mathrm{a}$ & $4.09 a$ & $4.35 \mathrm{a}$ \\
\hline & 3.0 & $2.59 \mathrm{a}$ & $2.35 \mathrm{a}$ & $2.97 \mathrm{a}$ & $4.24 \mathrm{a}$ & $2.91 \mathrm{a}$ & $2.53 \mathrm{a}$ \\
\hline \multirow{4}{*}{ Dry shoot weight (g) } & 0.0 & $0.62 \mathrm{a}$ & $0.62 \mathrm{a}$ & $0.62 \mathrm{a}$ & $0.62 \mathrm{a}$ & $0.62 \mathrm{a}$ & $0.62 \mathrm{a}$ \\
\hline & 1.0 & $0.66 \mathrm{a}$ & $0.54 \mathrm{a}$ & $0.66 \mathrm{a}$ & $0.54 \mathrm{a}$ & $0.71 \mathrm{a}$ & $0.60 \mathrm{a}$ \\
\hline & 2.0 & $0.61 \mathrm{a}$ & $0.50 \mathrm{a}$ & $0.38 \mathrm{a}$ & $0.59 \mathrm{a}$ & $0.60 \mathrm{a}$ & $0.59 \mathrm{a}$ \\
\hline & 3.0 & $0.31 \mathrm{a}$ & $0.42 \mathrm{a}$ & $0.47 \mathrm{a}$ & $0.61 \mathrm{a}$ & $0.49 \mathrm{a}$ & $0.39 \mathrm{a}$ \\
\hline \multirow{4}{*}{ Fresh root weight (g) } & 0.0 & $6.56 \mathrm{a}$ & $6.56 a$ & $6.56 a$ & $6.56 \mathrm{a}$ & $6.56 \mathrm{a}$ & $6.56 a$ \\
\hline & 1.0 & $7.00 \mathrm{a}$ & $5.79 \mathrm{a}$ & $7.25 \mathrm{a}$ & $6.35 \mathrm{a}$ & $6.52 \mathrm{a}$ & $6.07 \mathrm{a}$ \\
\hline & 2.0 & $5.15 \mathrm{a}$ & $4.72 \mathrm{a}$ & $4.93 a$ & $6.65 a$ & 5.46a & $6.60 \mathrm{a}$ \\
\hline & 3.0 & $5.16 \mathrm{a}$ & $4.38 \mathrm{a}$ & $5.26 \mathrm{a}$ & $7.56 \mathrm{a}$ & $4.50 \mathrm{a}$ & $4.59 \mathrm{a}$ \\
\hline \multirow{4}{*}{ Dry root weight (g) } & 0.0 & $0.77 \mathrm{a}$ & $0.77 \mathrm{a}$ & $0.77 \mathrm{a}$ & $0.77 \mathrm{a}$ & $0.77 \mathrm{a}$ & $0.77 \mathrm{a}$ \\
\hline & 1.0 & $0.73 a$ & $0.60 \mathrm{a}$ & $0.74 \mathrm{a}$ & $0.62 \mathrm{a}$ & $0.71 \mathrm{a}$ & $0.74 \mathrm{a}$ \\
\hline & 2.0 & $0.56 \mathrm{a}$ & $0.71 \mathrm{a}$ & $0.65 a$ & $0.60 \mathrm{a}$ & $0.88 \mathrm{a}$ & $0.92 \mathrm{a}$ \\
\hline & 3.0 & $0.71 \mathrm{a}$ & $0.78 \mathrm{a}$ & $0.65 \mathrm{a}$ & $0.81 \mathrm{a}$ & $0.88 \mathrm{a}$ & $0.74 \mathrm{a}$ \\
\hline \multirow{4}{*}{$\mathrm{F}_{\mathrm{v}} / \mathrm{F}_{\mathrm{m}}$} & 0.0 & $0.67 \mathrm{abc}$ & $0.67 \mathrm{abc}$ & $0.67 \mathrm{abc}$ & $0.67 \mathrm{abc}$ & $0.67 \mathrm{abc}$ & $0.67 \mathrm{abc}$ \\
\hline & 1.0 & $0.67 \mathrm{abc}$ & $0.65 \mathrm{bcd}$ & 0.64cde & $0.68 \mathrm{ab}$ & $0.71 \mathrm{a}$ & $0.60 \mathrm{~d}$ \\
\hline & 2.0 & $0.66 \mathrm{bcd}$ & $0.66 \mathrm{bcd}$ & $0.68 \mathrm{ab}$ & $0.68 \mathrm{ab}$ & $0.68 \mathrm{ab}$ & $0.62 \mathrm{~d}$ \\
\hline & 3.0 & $0.66 \mathrm{bcd}$ & $0.65 \mathrm{bcd}$ & $0.66 \mathrm{bcd}$ & $0.71 \mathrm{a}$ & 0.64 cde & $0.55 \mathrm{f}$ \\
\hline
\end{tabular}

Table 2. The interaction effects of $S \times L$ on I-29 faba bean genotype. ${ }^{x}$ Each value is the mean of three replications. ${ }^{y}$ Within salt source and salinity level of each parameter, means followed by the same letter are not significantly different at the $5 \%$ level according to Duncan's multiple range tests.

weight (ranged between 0.56 and $0.92 \mathrm{~g}$ ) parameters were not affected from $\mathrm{S} \times \mathrm{L}$ interaction (Table 2). None of the emergence percentage values starting from on the 9th DAS until the experiment lasted was not statistically significant. As an indication of photosynthetic capacity, the lowest $\mathrm{F}_{\mathrm{v}} / \mathrm{F}_{\mathrm{m}}$ ratio was observed under low, medium and high salinity levels with $\mathrm{Na}_{2} \mathrm{SO}_{4}$ salt source, however $\mathrm{F}_{\mathrm{v}} / \mathrm{F}_{\mathrm{m}}$ ratios under all salinity levels with other salt sources did not show significant difference from that of the control salinity level (Table 2). 
Performance of III-1 faba bean genotype. Emergence percentage at 24th DAS, mean emergence time and dry root wieght of the III-1 faba bean genotype were ranged from 70.0 to $100.0 \%$, from 9.31 to 12.69 days and from 0.40 to $0.68 \mathrm{~g}$, respectively, but there was no statistically significant difference among these values under $\mathrm{S} \times \mathrm{L}$ interaction. Throughout the experimental period, only the emergence percentage at 9 th and 10th DAS showed significant difference. Shoot height of this genotype were significantly decreased under each increasing salinity levels with all salt sources. In general, medium and high salinity levels with $\mathrm{CaCl}_{2}$ and $\mathrm{Na}_{2} \mathrm{SO}_{4}$, and high salinity level with $\mathrm{MgCl}_{2}, \mathrm{NaCl}$ and $\mathrm{MgSO}_{4}$ salt sources caused progressive decreases on fresh biomass, shoot and root weight whereas no effect of the $\mathrm{CaSO}_{4}$ salt under all salinity level was observed on these parameters. Similarly, medium and high salinity levels with $\mathrm{CaCl}_{2}$ and high salinity level with $\mathrm{Na}$ containing salts; and medium and high salinity levels with $\mathrm{CaCl}_{2}, \mathrm{MgCl}_{2}$ and $\mathrm{Na}_{2} \mathrm{SO}_{4}$, and high salinity level with $\mathrm{NaCl}$ salt source caused significant reductions on dry biomass and shoot weights, respectively. However, both parameter values under all salinity levels with $\mathrm{CaSO}_{4}$ and $\mathrm{MgSO}_{4}$ in addition to $\mathrm{MgCl}_{2}$ (only for dry biomass weights) salt sources did not show statistical significance. Profoundly low $\mathrm{F}_{\mathrm{v}} / \mathrm{F}_{\mathrm{m}}$ ratios were observed under medium and high salinity levels with $\mathrm{Na}_{2} \mathrm{SO}_{4}$ salt source (Table 3).

Performance of III-22 faba bean genotype. Although emergence percentage at 10th DAS, shoot height, fresh shoot weight and dry root weight values were ranged between 0.0 and $30.0 \%, 3.60$ and $8.17 \mathrm{~cm}, 2.01$ and $4.23 \mathrm{~g}$ and 0.47 and $0.71 \mathrm{~g}$, respectively, there was no significant difference among these values under $\mathrm{S} \times \mathrm{L}$ interaction. Emergence percentage values after 12th DAS were significantly different. The lowest emergence percentage at 24th DAS was observed under high salinity level with $\mathrm{NaCl}$ salt source. The statistical results revealed that high salinity level with $\mathrm{CaCl}_{2}$, medium and high salinity levels with $\mathrm{NaCl}$ salt sources significantly retarded the mean emergence time of III-2 seeds. Compared to control treatment, progresive decreases on fresh biomass weight under high salinity level with $\mathrm{CaCl}_{2}$, and $\mathrm{NaCl}$, and under medium and high salinity levels with $\mathrm{MgCl}_{2}$ salt sources; on dry biomass weight under high salinity level with $\mathrm{Na}$ containing salts; on dry shoot weight under high salinity level with all $\mathrm{Cl}$ containing salts; and on fresh root weight under high salinity level with $\mathrm{CaCl}_{2}$ and medium and high salinity levels with $\mathrm{MgCl}_{2}$ and $\mathrm{NaCl}$ salt sources. Similar to III- 1 , slightly low $\mathrm{F}_{\mathrm{v}} / \mathrm{F}_{\mathrm{m}}$ ratios were observed under medium and high salinity levels with $\mathrm{Na}_{2} \mathrm{SO}_{4}$ salt source (Table 4).

Performance of III-28 faba bean genotype. Considering $\mathrm{S} \times \mathrm{L}$ interaction, emergence percentage at 10th DAS, dry biomass weight, fresh shoot weight, dry shoot weight, dry root weight and $\mathrm{F}_{\mathrm{v}} / \mathrm{F}_{\mathrm{m}}$ ratio values were ranged between 0.0 and 26.7\%, 1.02 and $1.72 \mathrm{~g}, 2.14$ and $5.60 \mathrm{gr}, 0.43$ and $0.80 \mathrm{gr}, 0.51$ and $0.95 \mathrm{~g}$ and 0.62 and 0.70 , respectively. However, there was no significant difference among the values of these parameters. Emergence percentage values after 11th DAS were significantly different (except at 19th DAS). Compared to the control treatment, emergence percentages at 24th DAS under medium salinity level with Ca contaning salts, under low salinity level with $\mathrm{MgCl}$, under medium and high salinity levels with $\mathrm{NaCl}$, and under low and medium salinity levels with $\mathrm{MgSO}_{4}$ salt sources were significantly decreased. Similarly, high salinity level with $\mathrm{CaCl}_{2}$ and $\mathrm{MgCl}_{2}$, and medium salinity level with $\mathrm{NaCl}$ significantly retarded the mean emergence time of III- 28 seeds. Shoot height of the III-28 faba bean genotype were significantly decreased, in general, especially under medium (except with $\mathrm{CaSO}_{4}$ salt) and high salinity levels with all salt sources. In general, fresh biomass weights were drastically decreased under increasing all salinity levels with $\mathrm{CaCl}_{2}$ and $\mathrm{MgCl}_{2}$; under medium and high salinity levels with $\mathrm{Na}$ containing salts; and under high salinity level with $\mathrm{CaSO}_{4}$ and $\mathrm{MgSO}_{4}$ salt sources. Also, fresh root weights under increasing all salinity levels with $\mathrm{Ca}$ containing salts, and under medium and high salinity levels with $\mathrm{MaCl}_{2}, \mathrm{NaCl}$ and $\mathrm{MgSO}_{4}$ salt sources showed profound decreases (Table 5).

Performance of III-29 faba bean genotype. Emergence percentage at 24th DAS (ranged between 80.0 and $100.0 \%$ ), mean emergence time (ranged between 9.41 and 11.45 day) and dry shoot weight (ranged between 0.34 and $0.63 \mathrm{~g}$ ) values of III-29 faba bean genotip were not statistically significant under S $\times$ L interaction. Throughout the experimental period, only the emergence percentage at 9th, 10th, 11th and 12th DAS showed significant difference. Emergence percentage at 10th DAS was significantly decreased, under high salinity level with $\mathrm{CaCl}_{2}$, under medium and high salinity levels with $\mathrm{MgCl}_{2}$ and $\mathrm{NaCl}$, and under all increasing salinity levels with $\mathrm{CaSO}_{4}$ salt sources. In general, increasing salinity levels with all salt sources (except low salinity level with $\mathrm{CaCl}_{2}$ salt) resulted in decreases on shoot heights. Fresh biomass, shoot and root weights exhibited a marked decrease under medium and/or high salinity levels with $\mathrm{Cl}$ containing but not with $\mathrm{SO}_{4}$ containing salt sources. Decreases on dry biomass weights were observed only under high salinity level with $\mathrm{MgCl}_{2}$, and under medium and high salinity levels with $\mathrm{NaCl}$ salt sources. Similar to III-1 and III-22, slightly low $\mathrm{F}_{\mathrm{v}} / \mathrm{F}_{\mathrm{m}}$ ratios were observed under medium and high salinity levels with $\mathrm{Na}_{2} \mathrm{SO}_{4}$ salt source (Table 6).

Performance of USK-1 faba bean genotype. Emergence percentage values of the USK-1 faba bean genotype were ranged from 30.0 to $76.7 \%$ at 10 th DAS, and from 83.3 to $100.0 \%$ at 24 th DAS but there was no statistically significant difference among the values of these parameters under $\mathrm{S} \times \mathrm{L}$ interaction. Similarly, mean emergence time (ranged between 10.05 and 11.89 days) did not show a significant difference. Throughout the experimental period, only the emergence percentage at 9th DAS showed significant difference. Compared to control salinity level, shoot heights were significantly decreased especially under medium and high salinity levels with all salt sources. In general, compared to control, significant decreases were obtained for fresh biomass weights under medium and high salinity levels with all $\mathrm{Cl}$ containing salt sources and with $\mathrm{Na}_{2} \mathrm{SO}_{4}$ in addition to under high salinity level with $\mathrm{MgSO}_{4}$ salt source. Also, fresh shoot weights were negatively affected under high salinity level with $\mathrm{CaCl}_{2}, \mathrm{MgSO}_{4}$ and $\mathrm{Na}_{2} \mathrm{SO}_{4}$, under medium salinity level with $\mathrm{MgCl}_{2}$, under low salinity level $\mathrm{CaSO}_{4}$, and under medium and high salinity levels with $\mathrm{NaCl}$ salt sources. Similarly, medium and high salinity 


\begin{tabular}{|c|c|c|c|c|c|c|c|}
\hline \multirow[b]{2}{*}{ Parameter } & \multirow[b]{2}{*}{ Salinity level (dS/m) } & \multicolumn{6}{|l|}{ Salt source } \\
\hline & & $\mathrm{CaCl}_{2}$ & $\mathrm{MgCl}_{2}$ & $\mathrm{NaCl}$ & $\mathrm{CaSO}_{4}$ & $\mathrm{MgSO}_{4}$ & $\mathrm{Na}_{2} \mathrm{SO}_{4}$ \\
\hline \multirow{4}{*}{$\begin{array}{l}\text { Emergence percentage at 10th } \\
\text { DAS }\end{array}$} & 0.0 & $73.33^{x} a^{y}$ & 73.33ab & 73.33ab & 73.33ab & $73.33 \mathrm{ab}$ & $73.33 \mathrm{ab}$ \\
\hline & 1.0 & $46.67 \mathrm{bcdef}$ & 56.67abcde & 76.67ab & 63.33abc & 63.33abc & $83.33 a$ \\
\hline & 2.0 & $26.67 \mathrm{fg}$ & $33.33 \mathrm{efg}$ & $33.33 \mathrm{efg}$ & 73.33ab & $60.00 \mathrm{abcd}$ & $43.33 \mathrm{cdefg}$ \\
\hline & 3.0 & $20.00 \mathrm{~g}$ & $26.67 \mathrm{fg}$ & 36.67defg & 73.33ab & $36.67 \mathrm{defg}$ & $56.67 \mathrm{abcde}$ \\
\hline \multirow{4}{*}{$\begin{array}{l}\text { Emergence percentage at 24th } \\
\text { DAS }\end{array}$} & 0.0 & $86.67 \mathrm{a}$ & $86.67 \mathrm{a}$ & $86.67 \mathrm{a}$ & $86.67 \mathrm{a}$ & $86.67 \mathrm{a}$ & $86.67 \mathrm{a}$ \\
\hline & 1.0 & $90.00 \mathrm{a}$ & $96.67 \mathrm{a}$ & $100.00 \mathrm{a}$ & $90.00 \mathrm{a}$ & $80.00 \mathrm{a}$ & $86.67 \mathrm{a}$ \\
\hline & 2.0 & $90.00 \mathrm{a}$ & $96.67 \mathrm{a}$ & $86.67 \mathrm{a}$ & $83.33 a$ & $80.00 \mathrm{a}$ & $73.33 a$ \\
\hline & 3.0 & $83.33 a$ & $70.00 \mathrm{a}$ & $90.00 \mathrm{a}$ & $90.00 \mathrm{a}$ & $73.33 a$ & $86.67 \mathrm{a}$ \\
\hline \multirow{4}{*}{ Mean emergence time (day) } & 0.0 & $9.65 \mathrm{a}$ & $9.65 \mathrm{a}$ & $9.65 \mathrm{a}$ & $9.65 \mathrm{a}$ & $9.65 \mathrm{a}$ & $9.65 \mathrm{a}$ \\
\hline & 1.0 & $11.37 \mathrm{a}$ & $10.68 \mathrm{a}$ & $10.77 \mathrm{a}$ & $10.29 \mathrm{a}$ & $10.25 \mathrm{a}$ & $9.31 \mathrm{a}$ \\
\hline & 2.0 & $11.78 \mathrm{a}$ & $11.14 \mathrm{a}$ & $11.47 \mathrm{a}$ & $9.54 \mathrm{a}$ & $11.01 \mathrm{a}$ & $10.15 a$ \\
\hline & 3.0 & $12.69 \mathrm{a}$ & $11.18 \mathrm{a}$ & $11.71 \mathrm{a}$ & $10.31 \mathrm{a}$ & $11.02 \mathrm{a}$ & $10.14 a$ \\
\hline \multirow{4}{*}{ Shoot height (cm) } & 0.0 & $10.75 a$ & $10.75 a$ & $10.75 a$ & $10.75 a$ & $10.75 \mathrm{a}$ & $10.75 a$ \\
\hline & 1.0 & $7.58 \mathrm{bcd}$ & 7.06cde & $7.63 \mathrm{bcd}$ & $8.12 \mathrm{bcd}$ & $8.49 \mathrm{bc}$ & $8.01 \mathrm{bcd}$ \\
\hline & 2.0 & $4.34 \mathrm{fg}$ & 6.55de & $4.92 \mathrm{fg}$ & $8.32 \mathrm{bc}$ & $7.41 \mathrm{bcd}$ & $5.75 \mathrm{ef}$ \\
\hline & 3.0 & $4.00 \mathrm{~g}$ & $3.98 \mathrm{~g}$ & $3.88 \mathrm{~g}$ & $8.76 \mathrm{~b}$ & $6.67 \mathrm{de}$ & $4.57 \mathrm{fg}$ \\
\hline \multirow{4}{*}{ Fresh biomass weight (g) } & 0.0 & $9.66 \mathrm{abc}$ & $9.66 \mathrm{abc}$ & 9.66abc & $9.66 \mathrm{abc}$ & $9.66 \mathrm{abc}$ & $9.66 \mathrm{abc}$ \\
\hline & 1.0 & $9.05 \mathrm{abc}$ & $8.75 \mathrm{abcd}$ & 8.87abcd & $9.43 \mathrm{abc}$ & $10.46 \mathrm{ab}$ & $7.86 \mathrm{bcde}$ \\
\hline & 2.0 & $6.17 \mathrm{de}$ & $8.22 \mathrm{bcde}$ & 6.93cde & $11.62 \mathrm{a}$ & $7.12 \mathrm{cde}$ & $5.58 \mathrm{e}$ \\
\hline & 3.0 & 6.06de & $5.54 \mathrm{e}$ & $5.79 \mathrm{e}$ & $11.51 \mathrm{a}$ & $5.67 \mathrm{e}$ & $5.54 \mathrm{e}$ \\
\hline \multirow{4}{*}{ Dry biomass weight (g) } & 0.0 & $1.13 \mathrm{abcd}$ & $1.13 \mathrm{abcd}$ & $1.13 \mathrm{abcd}$ & $1.13 \mathrm{abcd}$ & $1.13 \mathrm{abcd}$ & $1.13 \mathrm{abcd}$ \\
\hline & 1.0 & $0.91 \mathrm{cde}$ & $1.02 \mathrm{bcde}$ & 0.89cde & $1.02 \mathrm{bcde}$ & $1.33 \mathrm{a}$ & $1.13 \mathrm{abcd}$ \\
\hline & 2.0 & $0.79 \mathrm{e}$ & $0.87 \mathrm{cde}$ & $0.96 \mathrm{cde}$ & $1.27 \mathrm{ab}$ & $1.16 \mathrm{abc}$ & $0.87 \mathrm{cde}$ \\
\hline & 3.0 & $0.74 \mathrm{e}$ & $0.84 \mathrm{de}$ & $0.78 \mathrm{e}$ & $1.27 \mathrm{ab}$ & $1.11 \mathrm{abcd}$ & $0.80 \mathrm{e}$ \\
\hline \multirow{4}{*}{ Fresh shoot weight (g) } & 0.0 & $4.44 \mathrm{ab}$ & $4.44 \mathrm{ab}$ & $4.44 \mathrm{ab}$ & $4.44 \mathrm{ab}$ & $4.44 \mathrm{ab}$ & $4.44 \mathrm{ab}$ \\
\hline & 1.0 & $4.16 \mathrm{abc}$ & $4.07 \mathrm{abc}$ & $3.85 \mathrm{abc}$ & $3.97 \mathrm{abc}$ & $4.68 \mathrm{a}$ & $3.98 \mathrm{abc}$ \\
\hline & 2.0 & $2.74 \mathrm{~d}$ & $3.94 \mathrm{abc}$ & $3.08 \mathrm{~cd}$ & $4.45 \mathrm{ab}$ & $3.35 \mathrm{bcd}$ & $2.49 \mathrm{~d}$ \\
\hline & 3.0 & $2.60 \mathrm{~d}$ & $2.25 \mathrm{~d}$ & $2.32 \mathrm{~d}$ & $4.89 \mathrm{a}$ & $2.59 \mathrm{~d}$ & $2.43 \mathrm{~d}$ \\
\hline \multirow{4}{*}{ Dry shoot weight (g) } & 0.0 & $0.59 \mathrm{ab}$ & $0.59 \mathrm{ab}$ & $0.59 \mathrm{ab}$ & $0.59 \mathrm{ab}$ & $0.59 \mathrm{ab}$ & $0.59 \mathrm{ab}$ \\
\hline & 1.0 & 0.52abcd & $0.55 \mathrm{abc}$ & 0.50abcde & 0.50abcde & $0.65 \mathrm{a}$ & $0.56 \mathrm{abc}$ \\
\hline & 2.0 & $0.37 \mathrm{ef}$ & $0.46 \mathrm{cdef}$ & 0.50abcde & $0.65 \mathrm{a}$ & $0.52 \mathrm{abcd}$ & $0.40 \mathrm{def}$ \\
\hline & 3.0 & $0.32 \mathrm{~g}$ & $0.34 \mathrm{fg}$ & $0.35 f g$ & $0.66 \mathrm{a}$ & $0.48 \mathrm{bcdef}$ & $0.37 \mathrm{ef}$ \\
\hline \multirow{4}{*}{ Fresh root weight (g) } & 0.0 & $5.23 \mathrm{abcd}$ & 5.23abcd & 5.23abcd & 5.23abcd & $5.23 \mathrm{abcd}$ & 5.23abcd \\
\hline & 1.0 & 4.89bcdef & 4.69bcdef & 5.02bcdef & 5.46abcd & $5.78 \mathrm{abc}$ & $3.88 \mathrm{cdef}$ \\
\hline & 2.0 & $3.43 \mathrm{ef}$ & $4.28 \mathrm{cdef}$ & $3.85 \mathrm{cdef}$ & $7.16 \mathrm{a}$ & $3.77 \mathrm{def}$ & $3.09 \mathrm{f}$ \\
\hline & 3.0 & $3.46 \mathrm{ef}$ & $3.30 \mathrm{ef}$ & $3.47 \mathrm{ef}$ & 6.62ab & $3.08 \mathrm{f}$ & $3.12 \mathrm{f}$ \\
\hline \multirow{4}{*}{ Dry root weight (g) } & 0.0 & $0.54 \mathrm{a}$ & $0.54 \mathrm{a}$ & $0.54 \mathrm{a}$ & $0.54 \mathrm{a}$ & $0.54 \mathrm{a}$ & $0.54 \mathrm{a}$ \\
\hline & 1.0 & $0.40 \mathrm{a}$ & $0.46 \mathrm{a}$ & $0.40 \mathrm{a}$ & $0.52 \mathrm{a}$ & $0.68 \mathrm{a}$ & $0.57 \mathrm{a}$ \\
\hline & 2.0 & $0.41 \mathrm{a}$ & $0.41 \mathrm{a}$ & $0.47 \mathrm{a}$ & $0.61 \mathrm{a}$ & $0.64 \mathrm{a}$ & $0.48 \mathrm{a}$ \\
\hline & 3.0 & $0.41 \mathrm{a}$ & $0.50 \mathrm{a}$ & $0.43 a$ & $0.61 \mathrm{a}$ & $0.62 \mathrm{a}$ & $0.43 a$ \\
\hline \multirow{4}{*}{$\mathrm{F}_{\mathrm{v}} / \mathrm{F}_{\mathrm{m}}$} & 0.0 & 0.66bcde & 0.66bcde & 0.66bcde & 0.66bcde & $0.66 \mathrm{bcde}$ & $0.66 \mathrm{bcde}$ \\
\hline & 1.0 & 0.65bcde & 0.67abcd & 0.65 bcde & $0.68 \mathrm{abc}$ & $0.70 \mathrm{a}$ & $0.61 \mathrm{ef}$ \\
\hline & 2.0 & 0.66bcde & $0.68 \mathrm{abc}$ & 0.66bcde & 0.66bcde & $0.69 \mathrm{ab}$ & $0.57 \mathrm{~g}$ \\
\hline & 3.0 & $0.69 \mathrm{ab}$ & $0.63 \mathrm{cde}$ & $0.65 \mathrm{bcde}$ & $0.69 \mathrm{ab}$ & $0.62 \mathrm{def}$ & $0.59 \mathrm{fg}$ \\
\hline
\end{tabular}

Table 3. The interaction effects of $S \times L$ on III- 1 faba bean genotype. ${ }^{x}$ Each value is the mean of three replications. ${ }^{y}$ Within salt source and salinity level of each parameter, means followed by the same letter are not significantly different at the $5 \%$ level according to Duncan's multiple range tests.

levels with all salt sources except $\mathrm{CaSO}_{4}$ and also low salinity level with $\mathrm{CaCl}_{2}$ cause significant reduction on fresh root weights. In general, marked decreases on dry biomass weights under medium or high salinity levels with all $\mathrm{Cl}$ containing salts and dry shoot and root weights under medium and/or high salinity levels with $\mathrm{CaCl}_{2}$ and $\mathrm{NaCl}$ salt sources were observed. In contrast, none of the salinity level with $\mathrm{SO}_{4}$ containing salt sources have an adverse effect on these dry weight parameters. The $\mathrm{F}_{\mathrm{v}} / \mathrm{F}_{\mathrm{m}}$ ratio was ranged between 0.58 and 0.72 . High salinity levels with $\mathrm{CaSO}_{4}$, and low and medium salinity levels with $\mathrm{MgSO}_{4}$ resulted in an increase on $\mathrm{F}_{\mathrm{v}} / \mathrm{F}_{\mathrm{m}}$ ratios whereas the lowest $\mathrm{F}_{\mathrm{v}} / \mathrm{F}_{\mathrm{m}}$ ratio was observed under high salinity level with $\mathrm{Na}_{2} \mathrm{SO}_{4}$ salt source (Table 7). 


\begin{tabular}{|c|c|c|c|c|c|c|c|}
\hline \multirow[b]{2}{*}{ Parameter } & \multirow[b]{2}{*}{ Salinity level (dS/m) } & \multicolumn{6}{|l|}{ Salt source } \\
\hline & & $\mathrm{CaCl}_{2}$ & $\mathrm{MgCl}_{2}$ & $\mathrm{NaCl}$ & $\mathrm{CaSO}_{4}$ & $\mathrm{MgSO}_{4}$ & $\mathrm{Na}_{2} \mathrm{SO}_{4}$ \\
\hline \multirow{4}{*}{ Emergence percentage at 10th DAS } & 0.0 & $16.67^{x} \mathrm{a}^{y}$ & $16.67 \mathrm{a}$ & $16.67 \mathrm{a}$ & $16.67 \mathrm{a}$ & $16.67 \mathrm{a}$ & $16.67 \mathrm{a}$ \\
\hline & 1.0 & $16.67 \mathrm{a}$ & $26.67 \mathrm{a}$ & $6.67 \mathrm{a}$ & $6.67 \mathrm{a}$ & $23.33 \mathrm{a}$ & $30.00 \mathrm{a}$ \\
\hline & 2.0 & $6.67 \mathrm{a}$ & $6.67 \mathrm{a}$ & $0.00 \mathrm{a}$ & $13.33 \mathrm{a}$ & $26.67 \mathrm{a}$ & $30.00 \mathrm{a}$ \\
\hline & 3.0 & $0.00 \mathrm{a}$ & $6.67 \mathrm{a}$ & $0.00 \mathrm{a}$ & $16.67 \mathrm{a}$ & $20.00 \mathrm{a}$ & $30.00 \mathrm{a}$ \\
\hline \multirow{4}{*}{ Emergence percentage at 24th DAS } & 0.0 & $96.67 \mathrm{ab}$ & $96.67 \mathrm{ab}$ & $96.67 \mathrm{ab}$ & $96.67 \mathrm{ab}$ & $96.67 \mathrm{ab}$ & $96.67 \mathrm{ab}$ \\
\hline & 1.0 & $93.33 \mathrm{ab}$ & $96.67 \mathrm{ab}$ & $93.33 \mathrm{ab}$ & $93.33 \mathrm{ab}$ & $96.67 \mathrm{ab}$ & $100.00 \mathrm{a}$ \\
\hline & 2.0 & $100.00 \mathrm{a}$ & $86.67 \mathrm{bc}$ & $100.00 \mathrm{a}$ & 93.33ab & $90.00 \mathrm{abc}$ & $100.00 \mathrm{a}$ \\
\hline & 3.0 & 90.00abc & $100.00 \mathrm{a}$ & $80.00 \mathrm{c}$ & 90.00abc & $100.00 \mathrm{a}$ & $100.00 \mathrm{a}$ \\
\hline \multirow{4}{*}{ Mean emergence time (day) } & 0.0 & $11.79 \mathrm{cde}$ & 11.79cde & 11.79cde & $11.79 \mathrm{cde}$ & $11.79 \mathrm{cde}$ & $11.79 \mathrm{cde}$ \\
\hline & 1.0 & $12.57 \mathrm{bcd}$ & 11.51cde & $12.62 \mathrm{bcd}$ & 11.97bcde & $11.83 \mathrm{cde}$ & $11.57 \mathrm{cde}$ \\
\hline & 2.0 & 12.10bcde & 12.19bcde & 13.30ab & 11.97bcde & $11.75 \mathrm{cde}$ & 11.30de \\
\hline & 3.0 & $14.17 \mathrm{a}$ & $12.87 \mathrm{bc}$ & $14.25 \mathrm{a}$ & $12.48 \mathrm{bcd}$ & $11.00 \mathrm{e}$ & $11.27 \mathrm{de}$ \\
\hline \multirow{4}{*}{ Shoot height $(\mathrm{cm})$} & 0.0 & $8.17 \mathrm{a}$ & $8.17 \mathrm{a}$ & $8.17 \mathrm{a}$ & $8.17 \mathrm{a}$ & $8.17 \mathrm{a}$ & $8.17 \mathrm{a}$ \\
\hline & 1.0 & $7.01 \mathrm{a}$ & $6.69 \mathrm{a}$ & 6.24a & $5.57 \mathrm{a}$ & $6.68 \mathrm{a}$ & $6.70 \mathrm{a}$ \\
\hline & 2.0 & $5.70 \mathrm{a}$ & $5.61 \mathrm{a}$ & $5.23 \mathrm{a}$ & $6.40 \mathrm{a}$ & $6.47 \mathrm{a}$ & $6.13 \mathrm{a}$ \\
\hline & 3.0 & $4.38 \mathrm{a}$ & $4.27 \mathrm{a}$ & $3.60 \mathrm{a}$ & $5.67 \mathrm{a}$ & $5.73 a$ & $4.70 \mathrm{a}$ \\
\hline \multirow{4}{*}{ Fresh biomass weight (g) } & 0.0 & $8.46 \mathrm{~cd}$ & $8.46 \mathrm{~cd}$ & $8.46 \mathrm{~cd}$ & $8.46 \mathrm{~cd}$ & $8.46 \mathrm{~cd}$ & $8.46 \mathrm{~cd}$ \\
\hline & 1.0 & 9.05abcd & $10.51 \mathrm{a}$ & $9.65 \mathrm{abc}$ & $8.20 \mathrm{~cd}$ & 9.06abcd & $10.16 \mathrm{ab}$ \\
\hline & 2.0 & $8.28 \mathrm{~cd}$ & 6.61ef & 7.56de & $8.93 \mathrm{bcd}$ & $9.68 \mathrm{abc}$ & $10.31 \mathrm{ab}$ \\
\hline & 3.0 & $5.76 \mathrm{fg}$ & $5.76 \mathrm{fg}$ & $4.43 \mathrm{~g}$ & $8.05 \mathrm{~d}$ & $8.42 \mathrm{~cd}$ & $8.35 \mathrm{~cd}$ \\
\hline \multirow{4}{*}{ Dry biomass weight (g) } & 0.0 & $1.16 \mathrm{bcd}$ & $1.16 \mathrm{bcd}$ & $1.16 \mathrm{bcd}$ & $1.16 \mathrm{bcd}$ & $1.16 \mathrm{bcd}$ & $1.16 \mathrm{bcd}$ \\
\hline & 1.0 & $1.19 \mathrm{ab}$ & $1.37 \mathrm{a}$ & $1.16 \mathrm{bcd}$ & $1.17 \mathrm{bc}$ & $1.16 \mathrm{bcd}$ & $1.13 \mathrm{bcd}$ \\
\hline & 2.0 & 1.10bcde & $1.11 \mathrm{bcde}$ & 0.99cdef & $1.22 \mathrm{ab}$ & $1.24 \mathrm{ab}$ & $1.12 \mathrm{bcde}$ \\
\hline & 3.0 & 0.96def & $0.98 \mathrm{cdef}$ & $0.85 f$ & $1.22 \mathrm{ab}$ & $1.12 \mathrm{bcde}$ & $0.93 \mathrm{ef}$ \\
\hline \multirow{4}{*}{ Fresh shoot weight (g) } & 0.0 & $3.66 \mathrm{a}$ & $3.66 \mathrm{a}$ & $3.66 \mathrm{a}$ & $3.66 \mathrm{a}$ & $3.66 \mathrm{a}$ & $3.66 \mathrm{a}$ \\
\hline & 1.0 & $4.21 \mathrm{a}$ & $4.11 \mathrm{a}$ & $3.83 \mathrm{a}$ & $3.54 \mathrm{a}$ & $3.89 \mathrm{a}$ & $4.23 \mathrm{a}$ \\
\hline & 2.0 & $3.68 \mathrm{a}$ & $3.08 \mathrm{a}$ & $3.54 \mathrm{a}$ & $4.01 \mathrm{a}$ & $4.03 a$ & $4.17 \mathrm{a}$ \\
\hline & 3.0 & $2.47 \mathrm{a}$ & $2.60 \mathrm{a}$ & $2.01 \mathrm{a}$ & $3.20 \mathrm{a}$ & $3.27 \mathrm{a}$ & $3.19 \mathrm{a}$ \\
\hline \multirow{4}{*}{ Dry shoot weight (g) } & 0.0 & $0.51 \mathrm{bcde}$ & $0.51 \mathrm{bcde}$ & $0.51 \mathrm{bcde}$ & $0.51 \mathrm{bcde}$ & $0.51 \mathrm{bcde}$ & $0.51 \mathrm{bcde}$ \\
\hline & 1.0 & 0.56abcd & $0.66 \mathrm{a}$ & 0.54abcd & 0.54abcd & $0.57 \mathrm{abcd}$ & $0.54 \mathrm{abcd}$ \\
\hline & 2.0 & 0.48 cdef & $0.55 \mathrm{abcd}$ & $0.45 \mathrm{def}$ & $0.63 \mathrm{ab}$ & $0.65 \mathrm{ab}$ & $0.60 \mathrm{abc}$ \\
\hline & 3.0 & $0.37 \mathrm{fg}$ & $0.38 \mathrm{fg}$ & $0.32 \mathrm{~g}$ & 0.54abcd & $0.55 \mathrm{abcd}$ & 0.45 def \\
\hline \multirow{4}{*}{ Fresh root weight (g) } & 0.0 & $4.81 \mathrm{de}$ & $4.81 \mathrm{de}$ & $4.81 \mathrm{de}$ & $4.81 \mathrm{de}$ & $4.81 \mathrm{de}$ & $4.81 \mathrm{de}$ \\
\hline & 1.0 & 4.84de & $6.40 \mathrm{a}$ & $5.82 \mathrm{ab}$ & $4.65 \mathrm{de}$ & $5.17 \mathrm{bcd}$ & $5.93 \mathrm{ab}$ \\
\hline & 2.0 & 4.60de & $3.53 \mathrm{fg}$ & $4.02 \mathrm{ef}$ & $4.92 \mathrm{~cd}$ & $5.65 \mathrm{abc}$ & $6.13 a$ \\
\hline & 3.0 & $3.28 \mathrm{fg}$ & $3.16 \mathrm{~g}$ & $2.42 \mathrm{~h}$ & $4.85 \mathrm{de}$ & $5.15 \mathrm{bcd}$ & $5.16 \mathrm{bcd}$ \\
\hline \multirow{4}{*}{ Dry root weight (g) } & 0.0 & $0.65 \mathrm{a}$ & $0.65 a$ & $0.65 \mathrm{a}$ & $0.65 \mathrm{a}$ & $0.65 \mathrm{a}$ & $0.65 \mathrm{a}$ \\
\hline & 1.0 & $0.63 \mathrm{a}$ & $0.71 \mathrm{a}$ & $0.62 \mathrm{a}$ & $0.63 \mathrm{a}$ & $0.58 \mathrm{a}$ & $0.60 \mathrm{a}$ \\
\hline & 2.0 & $0.61 \mathrm{a}$ & $0.55 a$ & $0.53 a$ & $0.59 \mathrm{a}$ & $0.59 \mathrm{a}$ & $0.52 \mathrm{a}$ \\
\hline & 3.0 & $0.59 \mathrm{a}$ & $0.60 \mathrm{a}$ & $0.52 \mathrm{a}$ & $0.68 \mathrm{a}$ & $0.57 \mathrm{a}$ & $0.47 \mathrm{a}$ \\
\hline \multirow{4}{*}{$\mathrm{F}_{\mathrm{v}} / \mathrm{F}_{\mathrm{m}}$} & 0.0 & $0.66 \mathrm{bcd}$ & $0.66 \mathrm{bcd}$ & $0.66 \mathrm{bcd}$ & $0.66 \mathrm{bcd}$ & $0.66 \mathrm{bcd}$ & $0.66 \mathrm{bcd}$ \\
\hline & 1.0 & $0.67 \mathrm{abc}$ & $0.66 \mathrm{bcd}$ & $0.64 \mathrm{cde}$ & $0.68 \mathrm{abc}$ & $0.70 \mathrm{a}$ & $0.63 \mathrm{def}$ \\
\hline & 2.0 & $0.67 \mathrm{abc}$ & $0.67 \mathrm{abc}$ & $0.65 \mathrm{cde}$ & $0.66 \mathrm{bcd}$ & $0.68 \mathrm{abc}$ & $0.61 \mathrm{ef}$ \\
\hline & 3.0 & $0.66 \mathrm{bcd}$ & $0.65 \mathrm{cde}$ & $0.65 \mathrm{cde}$ & $0.70 \mathrm{a}$ & $0.63 \mathrm{def}$ & $0.60 f$ \\
\hline
\end{tabular}

Table 4. The interaction effects of $S \times \mathrm{L}$ on III-22 faba bean genotype. ${ }^{\mathrm{x}}$ Each value is the mean of three replications. ${ }^{y}$ Within salt source and salinity level of each parameter, means followed by the same letter are not significantly different at the $5 \%$ level according to Duncan's multiple range tests.

Comparison of faba bean genotypes under the main effect of salinity levels and salt sources. Considering all salinity levels, the lowest and the highest values were obtained, in general, from faba bean genotypes of; I-29/III-28 and III-1/III-29 for emergence percentage at 10th DAS, I-29/III-1/III-28 and III-22 for emergence percentage at 24th DAS, III-1/III-29 and I-29/III-22 for mean emergence time, III-22/III29 and I-29/III-1 for shoot height, III-1/III-22 and I-29/III-28 for fresh biomass weight, USK-1/III-1 and I-29/ III-28 for dry biomass weight, USK-1/III-1/III-22 and I-29/III-28/III-29 for fresh shoot weight, USK-1/III-1/ 


\begin{tabular}{|c|c|c|c|c|c|c|c|}
\hline \multirow[b]{2}{*}{ Parameter } & \multirow[b]{2}{*}{ Salinity level (dS/m) } & \multicolumn{6}{|c|}{ Salt source } \\
\hline & & $\mathrm{CaCl}_{2}$ & $\mathrm{MgCl}_{2}$ & $\mathrm{NaCl}$ & $\mathrm{CaSO}_{4}$ & $\mathrm{MgSO}_{4}$ & $\mathrm{Na}_{2} \mathrm{SO}_{4}$ \\
\hline \multirow{4}{*}{ Emergence percentage at 10th DAS } & 0.0 & $13.33^{x} \mathrm{a}^{y}$ & $13.33 \mathrm{a}$ & $13.33 \mathrm{a}$ & $13.33 \mathrm{a}$ & $13.33 a$ & $13.33 a$ \\
\hline & 1.0 & $10.00 \mathrm{a}$ & $13.33 \mathrm{a}$ & $23.33 \mathrm{a}$ & $13.33 \mathrm{a}$ & $20.00 \mathrm{a}$ & $26.67 \mathrm{a}$ \\
\hline & 2.0 & $0.00 \mathrm{a}$ & $20.00 \mathrm{a}$ & $0.00 \mathrm{a}$ & $10.00 \mathrm{a}$ & $16.67 \mathrm{a}$ & $23.33 \mathrm{a}$ \\
\hline & 3.0 & $0.00 \mathrm{a}$ & $3.33 \mathrm{a}$ & $0.00 \mathrm{a}$ & $13.33 \mathrm{a}$ & $26.67 \mathrm{a}$ & $26.67 \mathrm{a}$ \\
\hline \multirow{4}{*}{ Emergence percentage at 24 th DAS } & 0.0 & $90.00 \mathrm{abc}$ & $90.00 \mathrm{abc}$ & $90.00 \mathrm{abc}$ & $90.00 \mathrm{abc}$ & $90.00 \mathrm{abc}$ & $90.00 \mathrm{abc}$ \\
\hline & 1.0 & 83.33cde & 80.00def & $90.00 \mathrm{abc}$ & $93.33 \mathrm{ab}$ & 80.00def & $86.67 \mathrm{bcd}$ \\
\hline & 2.0 & 80.00def & $96.67 \mathrm{a}$ & 80.00def & 76.67ef & 80.00def & $83.33 \mathrm{cde}$ \\
\hline & 3.0 & 93.33ab & $90.00 \mathrm{abc}$ & $73.33 \mathrm{f}$ & $86.67 \mathrm{bcd}$ & 86.67bcd & $90.00 \mathrm{abc}$ \\
\hline \multirow{4}{*}{ Mean emergence time (day) } & 0.0 & $11.67 \mathrm{de}$ & $11.67 \mathrm{de}$ & $11.67 \mathrm{de}$ & $11.67 \mathrm{de}$ & $11.67 \mathrm{de}$ & $11.67 \mathrm{de}$ \\
\hline & 1.0 & 11.96de & $12.25 \mathrm{cde}$ & 12.63bcde & 12.16cde & 11.71de & $11.25 \mathrm{e}$ \\
\hline & 2.0 & $13.14 \mathrm{bcd}$ & 11.71de & $13.84 \mathrm{abc}$ & 11.48de & $11.59 \mathrm{de}$ & $12.08 \mathrm{de}$ \\
\hline & 3.0 & $15.28 \mathrm{a}$ & 13.96ab & 12.91bcde & $11.77 \mathrm{de}$ & $12.27 \mathrm{cde}$ & $12.26 \mathrm{cde}$ \\
\hline \multirow{4}{*}{ Shoot height $(\mathrm{cm})$} & 0.0 & $9.40 \mathrm{ab}$ & $9.40 \mathrm{ab}$ & $9.40 \mathrm{ab}$ & $9.40 \mathrm{ab}$ & $9.40 \mathrm{ab}$ & $9.40 \mathrm{ab}$ \\
\hline & 1.0 & $8.10 \mathrm{c}$ & 6.63def & 6.98cde & $9.48 \mathrm{a}$ & $8.08 \mathrm{c}$ & 6.88cde \\
\hline & 2.0 & 4.79hi & 5.55fghi & 5.54fghi & $8.13 \mathrm{bc}$ & 6.88cde & 6.24defg \\
\hline & 3.0 & $3.45 j$ & $4.32 \mathrm{ij}$ & $3.54 \mathrm{j}$ & $7.40 \mathrm{~cd}$ & 5.76efgh & 5.11ghi \\
\hline \multirow{4}{*}{ Fresh biomass weight (g) } & 0.0 & $12.05 \mathrm{a}$ & $12.05 \mathrm{a}$ & $12.05 \mathrm{a}$ & $12.05 \mathrm{a}$ & $12.05 \mathrm{a}$ & $12.05 \mathrm{a}$ \\
\hline & 1.0 & $9.37 \mathrm{bcd}$ & $9.52 \mathrm{bcd}$ & $11.27 \mathrm{ab}$ & 9.59bcd & $10.25 \mathrm{abc}$ & $10.27 \mathrm{abc}$ \\
\hline & 2.0 & 7.51def & 7.03ef & 8.11cde & $9.89 \mathrm{abc}$ & $9.99 \mathrm{abc}$ & 9.16bcd \\
\hline & 3.0 & $5.91 \mathrm{fg}$ & $5.97 \mathrm{fg}$ & $4.87 \mathrm{~g}$ & $9.82 \mathrm{bc}$ & $8.63 \mathrm{cde}$ & $9.44 \mathrm{bcd}$ \\
\hline \multirow{4}{*}{ Dry biomass weight (g) } & 0.0 & $1.72 \mathrm{a}$ & $1.72 \mathrm{a}$ & $1.72 \mathrm{a}$ & $1.72 \mathrm{a}$ & $1.72 \mathrm{a}$ & $1.72 \mathrm{a}$ \\
\hline & 1.0 & $1.35 \mathrm{a}$ & $1.24 \mathrm{a}$ & $1.47 \mathrm{a}$ & $1.38 \mathrm{a}$ & $1.38 \mathrm{a}$ & $1.18 \mathrm{a}$ \\
\hline & 2.0 & $1.17 \mathrm{a}$ & $1.12 \mathrm{a}$ & $1.11 \mathrm{a}$ & $1.49 \mathrm{a}$ & $1.30 \mathrm{a}$ & $1.10 \mathrm{a}$ \\
\hline & 3.0 & $1.09 \mathrm{a}$ & $1.02 \mathrm{a}$ & $1.03 \mathrm{a}$ & $1.44 \mathrm{a}$ & $1.23 \mathrm{a}$ & $1.10 \mathrm{a}$ \\
\hline \multirow{4}{*}{ Fresh shoot weight (g) } & 0.0 & $5.00 \mathrm{a}$ & $5.00 \mathrm{a}$ & $5.00 \mathrm{a}$ & $5.00 \mathrm{a}$ & $5.00 \mathrm{a}$ & $5.00 \mathrm{a}$ \\
\hline & 1.0 & $4.07 \mathrm{a}$ & $3.89 \mathrm{a}$ & $4.75 \mathrm{a}$ & $5.6 \mathrm{a}$ & $4.76 \mathrm{a}$ & $4.44 \mathrm{a}$ \\
\hline & 2.0 & $3.48 \mathrm{a}$ & $3.28 \mathrm{a}$ & $3.97 \mathrm{a}$ & $4.49 \mathrm{a}$ & $4.39 \mathrm{a}$ & $3.54 \mathrm{a}$ \\
\hline & 3.0 & $2.25 \mathrm{a}$ & $2.55 \mathrm{a}$ & $2.15 \mathrm{a}$ & $4.39 \mathrm{a}$ & $3.60 \mathrm{a}$ & $3.66 \mathrm{a}$ \\
\hline \multirow{4}{*}{ Dry shoot weight $(\mathrm{g})$} & 0.0 & $0.77 \mathrm{a}$ & $0.77 \mathrm{a}$ & $0.77 \mathrm{a}$ & $0.77 \mathrm{a}$ & $0.77 \mathrm{a}$ & $0.77 \mathrm{a}$ \\
\hline & 1.0 & $0.53 a$ & $0.54 \mathrm{a}$ & $0.67 \mathrm{a}$ & $0.80 \mathrm{a}$ & $0.68 \mathrm{a}$ & $0.62 \mathrm{a}$ \\
\hline & 2.0 & $0.53 a$ & $0.50 \mathrm{a}$ & $0.55 \mathrm{a}$ & $0.69 \mathrm{a}$ & $0.69 \mathrm{a}$ & $0.57 \mathrm{a}$ \\
\hline & 3.0 & $0.53 \mathrm{a}$ & $0.50 \mathrm{a}$ & $0.44 \mathrm{a}$ & $0.71 \mathrm{a}$ & $0.63 \mathrm{a}$ & $0.58 \mathrm{a}$ \\
\hline \multirow{4}{*}{ Fresh root weight (g) } & 0.0 & $7.05 \mathrm{a}$ & $7.05 \mathrm{a}$ & $7.05 \mathrm{a}$ & $7.05 \mathrm{a}$ & $7.05 \mathrm{a}$ & $7.05 \mathrm{a}$ \\
\hline & 1.0 & 5.30bcde & $5.63 \mathrm{abc}$ & $6.52 \mathrm{ab}$ & 3.99efg & $5.60 \mathrm{abc}$ & $5.83 \mathrm{ab}$ \\
\hline & 2.0 & 4.03defg & $3.75 \mathrm{fg}$ & 4.14cdefg & 5.40bcde & $5.49 \mathrm{bcd}$ & $5.61 \mathrm{abc}$ \\
\hline & 3.0 & $3.65 \mathrm{fg}$ & $3.41 \mathrm{~g}$ & $2.73 \mathrm{~g}$ & 5.43bcde & 5.03bcdef & 5.78ab \\
\hline \multirow{4}{*}{ Dry root weight (g) } & 0.0 & $0.95 \mathrm{a}$ & $0.95 \mathrm{a}$ & $0.95 \mathrm{a}$ & $0.95 \mathrm{a}$ & $0.95 \mathrm{a}$ & $0.95 \mathrm{a}$ \\
\hline & 1.0 & $0.82 \mathrm{a}$ & $0.70 \mathrm{a}$ & $0.80 \mathrm{a}$ & $0.58 \mathrm{a}$ & $0.70 \mathrm{a}$ & $0.56 \mathrm{a}$ \\
\hline & 2.0 & $0.64 \mathrm{a}$ & $0.62 \mathrm{a}$ & $0.56 \mathrm{a}$ & $0.80 \mathrm{a}$ & $0.61 \mathrm{a}$ & $0.54 \mathrm{a}$ \\
\hline & 3.0 & $0.66 \mathrm{a}$ & $0.51 \mathrm{a}$ & $0.60 \mathrm{a}$ & $0.73 a$ & $0.59 \mathrm{a}$ & $0.52 \mathrm{a}$ \\
\hline \multirow{4}{*}{$\mathrm{F}_{\mathrm{v}} / \mathrm{F}_{\mathrm{m}}$} & 0.0 & $0.66 \mathrm{a}$ & $0.66 \mathrm{a}$ & $0.66 \mathrm{a}$ & $0.66 \mathrm{a}$ & $0.66 \mathrm{a}$ & $0.66 \mathrm{a}$ \\
\hline & 1.0 & $0.67 \mathrm{a}$ & $0.66 \mathrm{a}$ & $0.64 \mathrm{a}$ & $0.68 \mathrm{a}$ & $0.69 \mathrm{a}$ & $0.63 a$ \\
\hline & 2.0 & $0.67 \mathrm{a}$ & $0.67 \mathrm{a}$ & $0.64 \mathrm{a}$ & $0.66 \mathrm{a}$ & 0.70a & $0.63 \mathrm{a}$ \\
\hline & 3.0 & $0.62 \mathrm{a}$ & $0.64 \mathrm{a}$ & $0.65 \mathrm{a}$ & $0.69 \mathrm{a}$ & $0.63 a$ & $0.62 \mathrm{a}$ \\
\hline
\end{tabular}

Table 5. The interaction effects of $S \times \mathrm{L}$ on III-28 faba bean genotype. ${ }^{\mathrm{x}}$ Each value is the mean of three replications. ${ }^{\mathrm{y}}$ Within salt source and salinity level of each parameter, means followed by the same letter are not significantly different at the $5 \%$ level according to Duncan's multiple range tests.

III-22 and III28 for dry shoot weight, III-1/III-22/III-29 and I-29/III-28 for fresh root weight, III-1 and I-29/ III-28 for dry root weight, and I-29/III-1/III-28 and USK-1 for $\mathrm{F}_{\mathrm{v}} / \mathrm{F}_{\mathrm{m}}$ ratios (Fig. 1).

Similarly, considering all salt sources, in general, the lowest and the highest values were obtained from I-29/III-22/III-28 and III-1/III-29 for emergence percentage at 10th DAS, I-29/III-1/III-28 and III-22/III-29 for emergence percentage at 24th DAS, III-1/III-29 and I-29/III-22 for mean emergence time, III-22 and I-29/ USK-1/III-1 for shoot height, III-1/III-22/III-29 and I-29/III-28 for fresh biomass weight, USK-1/III-1/III-29 and I-29/III-28 for dry biomass weight, III-1/III-22 and I-29/III-28/III-29 for fresh shoot weight, USK-1/III-1/ 


\begin{tabular}{|c|c|c|c|c|c|c|c|}
\hline \multirow[b]{2}{*}{ Parameter } & \multirow[b]{2}{*}{ Salinity level (dS/m) } & \multicolumn{6}{|l|}{ Salt source } \\
\hline & & $\mathrm{CaCl}_{2}$ & $\mathrm{MgCl}_{2}$ & $\mathrm{NaCl}$ & $\mathrm{CaSO}_{4}$ & $\mathrm{MgSO}_{4}$ & $\mathrm{Na}_{2} \mathrm{SO}_{4}$ \\
\hline \multirow{4}{*}{$\begin{array}{l}\text { Emergence percentage at 10th } \\
\text { DAS }\end{array}$} & 0.0 & $70.00^{\mathrm{x}} \mathrm{abc}^{\mathrm{y}}$ & $70.00 \mathrm{abc}$ & $70.00 \mathrm{abc}$ & $70.00 \mathrm{abc}$ & $70.00 \mathrm{abc}$ & $70.00 \mathrm{abc}$ \\
\hline & 1.0 & $76.67 \mathrm{ab}$ & $60.00 \mathrm{bcdef}$ & 63.33bcde & 46.67 efg & 50.00defg & $70.00 \mathrm{abc}$ \\
\hline & 2.0 & 63.33bcde & 46.67 efg & $36.67 \mathrm{~g}$ & 50.00defg & $70.00 \mathrm{abc}$ & 56.67cdef \\
\hline & 3.0 & $33.33 \mathrm{~g}$ & $43.33 \mathrm{fg}$ & $46.67 \mathrm{efg}$ & $43.33 \mathrm{fg}$ & $66.67 \mathrm{bcd}$ & $86.67 \mathrm{a}$ \\
\hline \multirow{4}{*}{$\begin{array}{l}\text { Emergence percentage at } 24 \text { th } \\
\text { DAS }\end{array}$} & 0.0 & $90.00 \mathrm{a}$ & $90.00 \mathrm{a}$ & $90.00 \mathrm{a}$ & $90.00 \mathrm{a}$ & $90.00 \mathrm{a}$ & $90.00 \mathrm{a}$ \\
\hline & 1.0 & 93.33a & $93.33 \mathrm{a}$ & $96.67 \mathrm{a}$ & $83.33 a$ & $83.33 a$ & $93.33 \mathrm{a}$ \\
\hline & 2.0 & $96.67 \mathrm{a}$ & $90.00 \mathrm{a}$ & $93.33 \mathrm{a}$ & $93.33 \mathrm{a}$ & $96.67 \mathrm{a}$ & $86.67 \mathrm{a}$ \\
\hline & 3.0 & $93.33 \mathrm{a}$ & $86.67 \mathrm{a}$ & $93.33 \mathrm{a}$ & $80.00 \mathrm{a}$ & $100.00 \mathrm{a}$ & $96.67 \mathrm{a}$ \\
\hline \multirow{4}{*}{ Mean emergence time (day) } & 0.0 & $10.76 \mathrm{a}$ & $10.76 \mathrm{a}$ & $10.76 \mathrm{a}$ & $10.76 \mathrm{a}$ & $10.76 \mathrm{a}$ & $10.76 \mathrm{a}$ \\
\hline & 1.0 & $10.56 \mathrm{a}$ & $10.94 \mathrm{a}$ & $10.42 \mathrm{a}$ & $10.79 \mathrm{a}$ & $10.30 \mathrm{a}$ & $10.04 \mathrm{a}$ \\
\hline & 2.0 & $10.39 \mathrm{a}$ & $10.98 \mathrm{a}$ & $11.45 \mathrm{a}$ & $10.24 \mathrm{a}$ & $10.33 a$ & $10.72 \mathrm{a}$ \\
\hline & 3.0 & $11.27 \mathrm{a}$ & $10.88 \mathrm{a}$ & $10.98 \mathrm{a}$ & $10.94 \mathrm{a}$ & $10.13 a$ & $9.41 \mathrm{a}$ \\
\hline \multirow{4}{*}{ Shoot height (cm) } & 0.0 & $9.35 \mathrm{a}$ & $9.35 a$ & $9.35 \mathrm{a}$ & $9.35 \mathrm{a}$ & $9.35 \mathrm{a}$ & $9.35 \mathrm{a}$ \\
\hline & 1.0 & $9.08 \mathrm{a}$ & $7.21 \mathrm{bc}$ & $7.38 \mathrm{bc}$ & $6.47 \mathrm{cde}$ & $7.68 \mathrm{~b}$ & $7.40 \mathrm{bc}$ \\
\hline & 2.0 & 5.99de & 5.95de & $4.87 \mathrm{fg}$ & 6.14de & 6.21de & 5.51ef \\
\hline & 3.0 & 4.05gh & $4.43 \mathrm{gh}$ & $3.97 \mathrm{~h}$ & $6.66 \mathrm{~cd}$ & $6.07 \mathrm{de}$ & $5.75 \mathrm{def}$ \\
\hline \multirow{4}{*}{ Fresh biomass weight (g) } & 0.0 & 9.34abcd & 9.34abcd & 9.34abcd & 9.34abcd & 9.34abcd & 9.34abcd \\
\hline & 1.0 & $11.33 \mathrm{a}$ & $8.36 \mathrm{bcdef}$ & $10.27 \mathrm{ab}$ & 7.91cdefg & 8.46bcdef & $9.97 \mathrm{abc}$ \\
\hline & 2.0 & 7.34defgh & 6.93efgh & 6.75 fghi & 7.48defgh & $8.36 \mathrm{bcdef}$ & $8.89 \mathrm{bcde}$ \\
\hline & 3.0 & 5.65hi & 6.26ghi & $4.90 \mathrm{i}$ & $9.11 \mathrm{bcd}$ & $8.39 \mathrm{bcdef}$ & $9.09 \mathrm{bcd}$ \\
\hline \multirow{4}{*}{ Dry biomass weight (g) } & 0.0 & 1.13abcde & 1.13abcde & 1.13abcde & 1.13abcde & 1.13abcde & 1.13abcde \\
\hline & 1.0 & $1.28 \mathrm{ab}$ & $1.18 \mathrm{abcd}$ & $1.31 \mathrm{a}$ & $1.20 \mathrm{abc}$ & $1.08 \mathrm{cdefg}$ & $1.08 \mathrm{cdefg}$ \\
\hline & 2.0 & $1.05 \mathrm{cdefg}$ & $1.08 \mathrm{cdefg}$ & 0.94 fgh & 1.11bcdef & $1.11 \mathrm{bcdef}$ & $0.97 \mathrm{efgh}$ \\
\hline & 3.0 & 1.01 defg & 0.92gh & $0.83 \mathrm{~h}$ & $1.30 \mathrm{a}$ & $1.11 \mathrm{bcdef}$ & 0.96 efgh \\
\hline \multirow{4}{*}{ Fresh shoot weight (g) } & 0.0 & 4.22abcd & 4.22abcd & 4.22abcd & 4.22abcd & 4.22abcd & 4.22abcd \\
\hline & 1.0 & $4.99 \mathrm{a}$ & $4.41 \mathrm{abc}$ & $4.56 \mathrm{ab}$ & 3.81bcdef & 4.08abcde & $4.35 \mathrm{abc}$ \\
\hline & 2.0 & 3.68bcdef & 3.55cdefg & 3.21efgh & 3.56cdefg & $3.68 \mathrm{bcdef}$ & 3.36defgh \\
\hline & 3.0 & 2.71gh & 2.96 fgh & $2.49 \mathrm{~h}$ & $4.02 \mathrm{bcde}$ & $3.57 \mathrm{cdefg}$ & 3.51cdefg \\
\hline \multirow{4}{*}{ Dry shoot weight (g) } & 0.0 & $0.54 \mathrm{a}$ & $0.54 \mathrm{a}$ & $0.54 \mathrm{a}$ & $0.54 \mathrm{a}$ & $0.54 \mathrm{a}$ & $0.54 \mathrm{a}$ \\
\hline & 1.0 & $0.63 a$ & $0.60 \mathrm{a}$ & $0.63 \mathrm{a}$ & $0.59 \mathrm{a}$ & $0.61 \mathrm{a}$ & $0.62 \mathrm{a}$ \\
\hline & 2.0 & $0.59 \mathrm{a}$ & $0.52 \mathrm{a}$ & $0.47 \mathrm{a}$ & $0.56 \mathrm{a}$ & $0.51 \mathrm{a}$ & $0.55 \mathrm{a}$ \\
\hline & 3.0 & $0.44 \mathrm{a}$ & $0.43 a$ & $0.34 \mathrm{a}$ & $0.57 \mathrm{a}$ & $0.58 \mathrm{a}$ & $0.50 \mathrm{a}$ \\
\hline \multirow{4}{*}{ Fresh root weight (g) } & 0.0 & $5.12 \mathrm{bcd}$ & $5.12 \mathrm{bcd}$ & $5.12 \mathrm{bcd}$ & $5.12 \mathrm{bcd}$ & $5.12 \mathrm{bcd}$ & $5.12 \mathrm{bcd}$ \\
\hline & 1.0 & $6.34 \mathrm{a}$ & 3.96def & 5.71ab & $4.11 \mathrm{def}$ & $4.38 \mathrm{cdef}$ & $5.62 \mathrm{ab}$ \\
\hline & 2.0 & $3.66 \mathrm{efg}$ & 3.37 fgh & $3.53 \mathrm{fg}$ & 3.93def & $4.69 \mathrm{bcde}$ & $5.54 \mathrm{abc}$ \\
\hline & 3.0 & 2.93gh & $3.30 \mathrm{fgh}$ & $2.40 \mathrm{~h}$ & $5.08 \mathrm{bcd}$ & $4.81 \mathrm{bcde}$ & $5.57 \mathrm{abc}$ \\
\hline \multirow{4}{*}{ Dry root weight (g) } & 0.0 & $0.59 \mathrm{bcde}$ & $0.59 \mathrm{bcde}$ & $0.59 \mathrm{bcde}$ & $0.59 \mathrm{bcde}$ & $0.59 \mathrm{bcde}$ & $0.59 \mathrm{bcde}$ \\
\hline & 1.0 & $0.65 \mathrm{abc}$ & $0.57 \mathrm{bcde}$ & $0.68 \mathrm{ab}$ & $0.60 \mathrm{bcd}$ & $0.47 \mathrm{def}$ & $0.46 \mathrm{ef}$ \\
\hline & 2.0 & $0.47 \mathrm{def}$ & 0.57bcde & 0.48def & 0.56bcde & $0.59 \mathrm{bcde}$ & $0.42 \mathrm{f}$ \\
\hline & 3.0 & $0.58 \mathrm{bcde}$ & 0.49def & 0.49def & $0.73 a$ & 0.53 cdef & $0.46 \mathrm{ef}$ \\
\hline \multirow{4}{*}{$\mathrm{F}_{\mathrm{v}} / \mathrm{F}_{\mathrm{m}}$} & 0.0 & $0.66 \mathrm{~cd}$ & $0.66 \mathrm{~cd}$ & $0.66 \mathrm{~cd}$ & $0.66 \mathrm{~cd}$ & $0.66 \mathrm{~cd}$ & $0.66 \mathrm{~cd}$ \\
\hline & 1.0 & $0.66 \mathrm{~cd}$ & $0.66 \mathrm{~cd}$ & $0.66 \mathrm{~cd}$ & $0.68 \mathrm{bc}$ & 0.70ab & $0.63 \mathrm{de}$ \\
\hline & 2.0 & $0.66 \mathrm{~cd}$ & $0.68 \mathrm{bc}$ & $0.66 \mathrm{~cd}$ & $0.67 \mathrm{bc}$ & $0.66 \mathrm{~cd}$ & $0.60 \mathrm{f}$ \\
\hline & 3.0 & $0.66 \mathrm{~cd}$ & $0.63 \mathrm{de}$ & $0.65 \mathrm{~cd}$ & $0.72 \mathrm{a}$ & $0.62 \mathrm{ef}$ & $0.60 \mathrm{f}$ \\
\hline
\end{tabular}

Table 6. The interaction effects of $S \times \mathrm{L}$ level on III-29 faba bean genotype. ${ }^{\mathrm{x}}$ Each value is the mean of three replications. ${ }^{y}$ Within salt source and salinity level of each parameter, means followed by the same letter are not significantly different at the $5 \%$ level according to Duncan's multiple range tests.

III-22 and III-28 for dry shoot weight, III-1/III-29 and I-29/III-28 for fresh root weight, and USK-1/III-29 and I-29/III-28 for dry root weight values (Fig. 2).

Principle component analysis (PCA). According to PCA, three eigenvalues higher than 1 were found, but the first two components are presented in Fig. 3. A total of $71.0 \%$ of the phenotypic variance was explained, with the first component being $48.6 \%$ and the second component being $22.4 \%$. The genotype III-22 was the most stable ones. So, this genotype was localized near the origin of the biplot. The high salinity level ( $3 \mathrm{dS} / \mathrm{m})$ was 


\begin{tabular}{|c|c|c|c|c|c|c|c|}
\hline \multirow[b]{2}{*}{ Parameter } & \multirow[b]{2}{*}{ Salinity level (dS/m) } & \multicolumn{6}{|c|}{ Salt source } \\
\hline & & $\mathrm{CaCl}_{2}$ & $\mathrm{MgCl}_{2}$ & $\mathrm{NaCl}$ & $\mathrm{CaSO}_{4}$ & $\mathrm{MgSO}_{4}$ & $\mathrm{Na}_{2} \mathrm{SO}_{4}$ \\
\hline \multirow{4}{*}{$\begin{array}{l}\text { Emergence percentage at } \\
\text { 10th DAS }\end{array}$} & 0.0 & $60.00^{\mathrm{x}} \mathrm{a}^{y}$ & $60.00 \mathrm{a}$ & $60.00 \mathrm{a}$ & $60.00 \mathrm{a}$ & $60.00 \mathrm{a}$ & $60.00 \mathrm{a}$ \\
\hline & 1.0 & $56.67 \mathrm{a}$ & $46.67 \mathrm{a}$ & $73.33 a$ & $60.00 \mathrm{a}$ & $56.67 \mathrm{a}$ & $76.67 \mathrm{a}$ \\
\hline & 2.0 & $43.33 \mathrm{a}$ & $40.00 \mathrm{a}$ & $50.00 \mathrm{a}$ & $60.00 \mathrm{a}$ & $60.00 \mathrm{a}$ & $56.67 \mathrm{a}$ \\
\hline & 3.0 & $40.00 \mathrm{a}$ & $70.00 \mathrm{a}$ & $30.00 \mathrm{a}$ & $53.33 \mathrm{a}$ & $50.00 \mathrm{a}$ & $66.67 \mathrm{a}$ \\
\hline \multirow{4}{*}{$\begin{array}{l}\text { Emergence percentage at } \\
\text { 24th DAS }\end{array}$} & 0.0 & $90.00 \mathrm{a}$ & $90.00 \mathrm{a}$ & $90.00 \mathrm{a}$ & $90.00 \mathrm{a}$ & $90.00 \mathrm{a}$ & $90.00 \mathrm{a}$ \\
\hline & 1.0 & $93.33 a$ & $90.00 \mathrm{a}$ & $100.00 \mathrm{a}$ & $90.00 \mathrm{a}$ & $86.67 \mathrm{a}$ & $93.33 \mathrm{a}$ \\
\hline & 2.0 & $90.00 \mathrm{a}$ & $83.33 a$ & $93.33 a$ & $83.33 a$ & $86.67 \mathrm{a}$ & $96.67 \mathrm{a}$ \\
\hline & 3.0 & $86.67 \mathrm{a}$ & $93.33 a$ & $86.67 \mathrm{a}$ & $86.67 \mathrm{a}$ & $96.67 \mathrm{a}$ & $96.67 \mathrm{a}$ \\
\hline \multirow{4}{*}{ Mean emergence time (day) } & 0.0 & $10.73 a$ & $10.73 a$ & $10.73 a$ & $10.73 a$ & $10.73 \mathrm{a}$ & $10.73 a$ \\
\hline & 1.0 & $11.19 \mathrm{a}$ & $11.89 \mathrm{a}$ & $11.00 \mathrm{a}$ & $10.59 \mathrm{a}$ & $11.44 \mathrm{a}$ & $10.05 \mathrm{a}$ \\
\hline & 2.0 & $10.96 \mathrm{a}$ & $10.30 \mathrm{a}$ & $10.97 \mathrm{a}$ & $10.51 \mathrm{a}$ & $10.97 \mathrm{a}$ & $10.76 \mathrm{a}$ \\
\hline & 3.0 & $10.96 \mathrm{a}$ & $10.27 \mathrm{a}$ & $11.52 \mathrm{a}$ & $10.66 \mathrm{a}$ & $11.43 \mathrm{a}$ & $10.16 \mathrm{a}$ \\
\hline \multirow{4}{*}{ Shoot height (cm) } & 0.0 & $10.22 \mathrm{a}$ & $10.22 \mathrm{a}$ & $10.22 \mathrm{a}$ & $10.22 \mathrm{a}$ & $10.22 \mathrm{a}$ & $10.22 \mathrm{a}$ \\
\hline & 1.0 & $7.98 \mathrm{~b}$ & 6.01efgh & $7.57 \mathrm{bc}$ & 6.62cdef & $7.48 \mathrm{bcd}$ & $8.05 \mathrm{~b}$ \\
\hline & 2.0 & 6.33cdefg & 4.95hi & 4.91hi & 7.25bcde & 7.33bcde & 6.26cdefgh \\
\hline & 3.0 & $4.33 \mathrm{ij}$ & $5.79 \mathrm{fgh}$ & $3.53 j$ & 6.13defgh & 6.52cdef & $5.09 \mathrm{ghi}$ \\
\hline \multirow{4}{*}{ Fresh biomass weight (g) } & 0.0 & $10.12 \mathrm{a}$ & $10.12 \mathrm{a}$ & $10.12 \mathrm{a}$ & $10.12 \mathrm{a}$ & $10.12 \mathrm{a}$ & $10.12 \mathrm{a}$ \\
\hline & 1.0 & $8.85 \mathrm{abc}$ & $8.77 \mathrm{abc}$ & $9.96 \mathrm{a}$ & $8.66 \mathrm{abc}$ & $10.22 \mathrm{a}$ & 9.81ab \\
\hline & 2.0 & $7.75 \mathrm{~cd}$ & $6.08 \mathrm{ef}$ & 6.86def & 9.42ab & $8.68 \mathrm{abc}$ & $8.27 \mathrm{bcd}$ \\
\hline & 3.0 & 5.87ef & $7.66 \mathrm{~cd}$ & $5.39 \mathrm{f}$ & 9.64ab & 7.25cde & $7.71 \mathrm{~cd}$ \\
\hline \multirow{4}{*}{ Dry biomass weight (g) } & 0.0 & $1.13 \mathrm{bcd}$ & $1.13 \mathrm{bcd}$ & $1.13 \mathrm{bcd}$ & $1.13 \mathrm{bcd}$ & $1.13 \mathrm{bcd}$ & $1.13 \mathrm{bcd}$ \\
\hline & 1.0 & 0.91defg & $0.89 \mathrm{defg}$ & $0.97 \mathrm{cdef}$ & 0.97cdef & $1.17 \mathrm{bc}$ & $1.49 \mathrm{a}$ \\
\hline & 2.0 & 0.87defg & 0.91 defg & $0.72 \mathrm{~g}$ & 1.04cde & $1.35 \mathrm{ab}$ & $1.08 \mathrm{cde}$ \\
\hline & 3.0 & $0.74 \mathrm{fg}$ & 0.84 efg & $0.87 \mathrm{defg}$ & $1.10 \mathrm{~cd}$ & $1.20 \mathrm{bc}$ & $1.07 \mathrm{cde}$ \\
\hline \multirow{4}{*}{ Fresh shoot weight (g) } & 0.0 & $4.37 \mathrm{a}$ & $4.37 \mathrm{a}$ & $4.37 \mathrm{a}$ & $4.37 \mathrm{a}$ & $4.37 \mathrm{a}$ & $4.37 \mathrm{a}$ \\
\hline & 1.0 & $4.27 \mathrm{ab}$ & 3.64abcdef & $4.34 \mathrm{a}$ & 3.31cdefg & $4.06 \mathrm{abc}$ & $4.13 \mathrm{abc}$ \\
\hline & 2.0 & 3.75abcde & 2.87 efgh & 2.94defgh & 3.62abcdefg & 3.78abcd & 3.55abcdefg \\
\hline & 3.0 & $2.73 \mathrm{gf}$ & 3.51abcdefg & $2.18 \mathrm{~h}$ & 3.59abcdefg & 3.40bcdefg & $2.81 \mathrm{fgh}$ \\
\hline \multirow{4}{*}{ Dry shoot weight (g) } & 0.0 & $0.56 \mathrm{abc}$ & $0.56 \mathrm{abc}$ & $0.56 \mathrm{abc}$ & $0.56 \mathrm{abc}$ & $0.56 \mathrm{abc}$ & $0.56 \mathrm{abc}$ \\
\hline & 1.0 & $0.51 \mathrm{abcd}$ & $0.48 \mathrm{abcd}$ & $0.53 \mathrm{abc}$ & $0.46 \mathrm{bcd}$ & $0.55 \mathrm{abc}$ & $0.63 a$ \\
\hline & 2.0 & $0.54 \mathrm{abc}$ & $0.43 \mathrm{~cd}$ & $0.37 \mathrm{~d}$ & $0.47 \mathrm{bcd}$ & $0.59 \mathrm{ab}$ & 0.49abcd \\
\hline & 3.0 & $0.38 \mathrm{~d}$ & $0.41 \mathrm{~cd}$ & $0.53 \mathrm{abc}$ & $0.53 \mathrm{abc}$ & $0.54 \mathrm{abc}$ & $0.42 \mathrm{~cd}$ \\
\hline \multirow{4}{*}{ Fresh root weight (g) } & 0.0 & $5.75 \mathrm{abc}$ & $5.75 \mathrm{abc}$ & $5.75 \mathrm{abc}$ & $5.75 \mathrm{abc}$ & $5.75 \mathrm{abc}$ & $5.75 \mathrm{abc}$ \\
\hline & 1.0 & $4.58 \mathrm{efg}$ & $5.13 \mathrm{bcde}$ & $5.62 \mathrm{abcd}$ & 5.36abcde & $6.16 \mathrm{a}$ & $5.69 \mathrm{abc}$ \\
\hline & 2.0 & 4.00fghi & $3.22 \mathrm{hi}$ & 3.91ghi & $5.81 \mathrm{abc}$ & 4.89cdef & 4.73defg \\
\hline & 3.0 & $3.14 \mathrm{i}$ & $4.15 \mathrm{fgh}$ & $3.20 \mathrm{hi}$ & 6.06ab & 3.85ghi & $4.90 \mathrm{cdef}$ \\
\hline \multirow{4}{*}{ Dry root weight (g) } & 0.0 & 0.57cdef & $0.57 \mathrm{cdef}$ & $0.57 \mathrm{cdef}$ & 0.57cdef & 0.57cdef & \begin{tabular}{|l|l|}
$0.57 \mathrm{cdef}$ \\
\end{tabular} \\
\hline & 1.0 & 0.40fgh & 0.41 efgh & 0.44 efgh & $0.52 \mathrm{cdefg}$ & $0.63 \mathrm{bcd}$ & $0.86 a$ \\
\hline & 2.0 & $0.33 \mathrm{~h}$ & $0.47 \mathrm{defgh}$ & $0.35 \mathrm{gh}$ & 0.57cdef & $0.76 \mathrm{ab}$ & 0.59cde \\
\hline & 3.0 & $0.36 \mathrm{gh}$ & 0.43 efgh & $0.35 \mathrm{gh}$ & 0.57cdef & $0.66 \mathrm{bc}$ & $0.65 \mathrm{bcd}$ \\
\hline \multirow{4}{*}{$\mathrm{F}_{\mathrm{v}} / \mathrm{F}_{\mathrm{m}}$} & 0.0 & 0.65def & $0.65 \mathrm{def}$ & $0.65 \mathrm{def}$ & $0.65 \mathrm{def}$ & $0.65 \mathrm{def}$ & $0.65 \mathrm{def}$ \\
\hline & 1.0 & 0.67bcde & $0.68 \mathrm{abcd}$ & $0.65 \mathrm{def}$ & 0.66bcdef & $0.72 \mathrm{a}$ & $0.62 \mathrm{efg}$ \\
\hline & 2.0 & 0.64def & $0.66 \mathrm{bcdef}$ & $0.69 \mathrm{abcd}$ & $0.65 \mathrm{def}$ & 0.71ab & $0.63 \mathrm{ef}$ \\
\hline & 3.0 & $0.68 \mathrm{abcd}$ & $0.65 \mathrm{cde}$ & $0.62 \mathrm{efg}$ & $0.71 \mathrm{ab}$ & $0.68 \mathrm{abcd}$ & $0.58 \mathrm{~g}$ \\
\hline
\end{tabular}

Table 7. The interaction effects of $S \times L$ on USK-1 faba bean genotype. ${ }^{x}$ Each value is the mean of three replications. ${ }^{y}$ Within salt source and salinity level of each parameter, means followed by the same letter are not significantly different at the $5 \%$ level according to Duncan's multiple range tests.

positioned far from the origin in the biplot indicates that it largely affected the genotypes. The genotypes I-29 and III-28 had a specific respond to salt sources $\mathrm{CaSO}_{4}$ and $\mathrm{MgSO}_{4}$, while the genotypes III- 1 and USK-1 had a specific respond to salt sources $\mathrm{CaCl}_{2}, \mathrm{MgCl}_{2}$ and $\mathrm{NaCl}$. This can be explained by the fact that genotypes react as distinct from to different salt sources (Fig. 3). Also, it was determined that the parameter that best explains the salinity tolerance is $\mathrm{F}_{\mathrm{v}} / \mathrm{F}_{\mathrm{m}} \cdot \mathrm{F}_{\mathrm{v}} / \mathrm{F}_{\mathrm{m}}$ ratios showed a significant difference for I-29 and III-28 genotypes. Fresh root weight, dry shoot weight, fresh biomass weight, fresh shoot weight and shoot height were found to be positively and significantly correlated with each other and they were negatively correlated with mean emergence time. As 

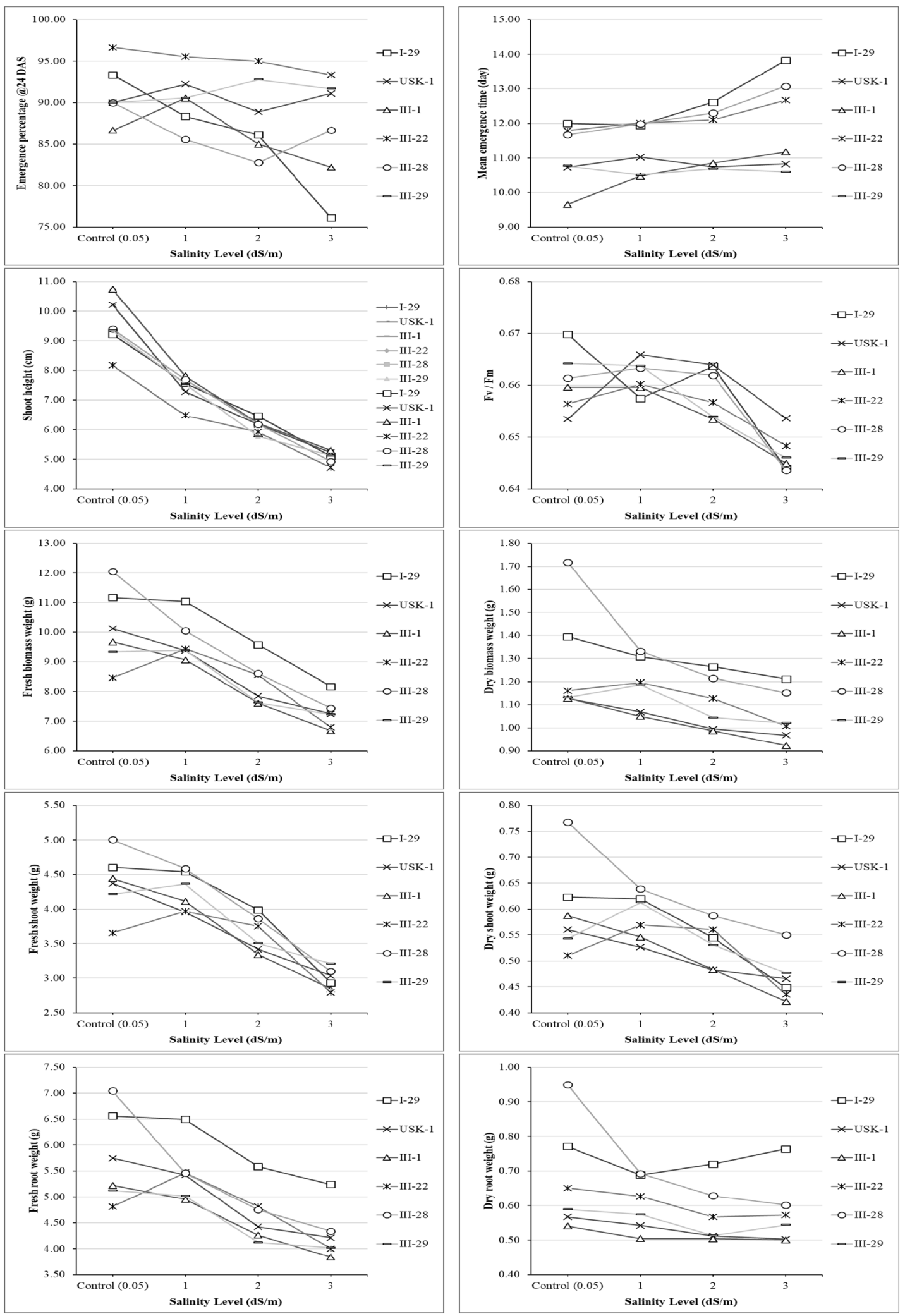

Figure 1. Comparison of faba bean genotypes under the main effect of salinity levels.

expected, these parameters decrease as mean emergence time increases. Dry root weight, dry biomass weight and $\mathrm{F}_{\mathrm{v}} / \mathrm{F}_{\mathrm{m}}$ parameters were positively correlated with each other. It was determined that I- 29 and III- 28 genotypes were better than other genotypes, especially in terms of dry root weight and dry biomass weight. While the 

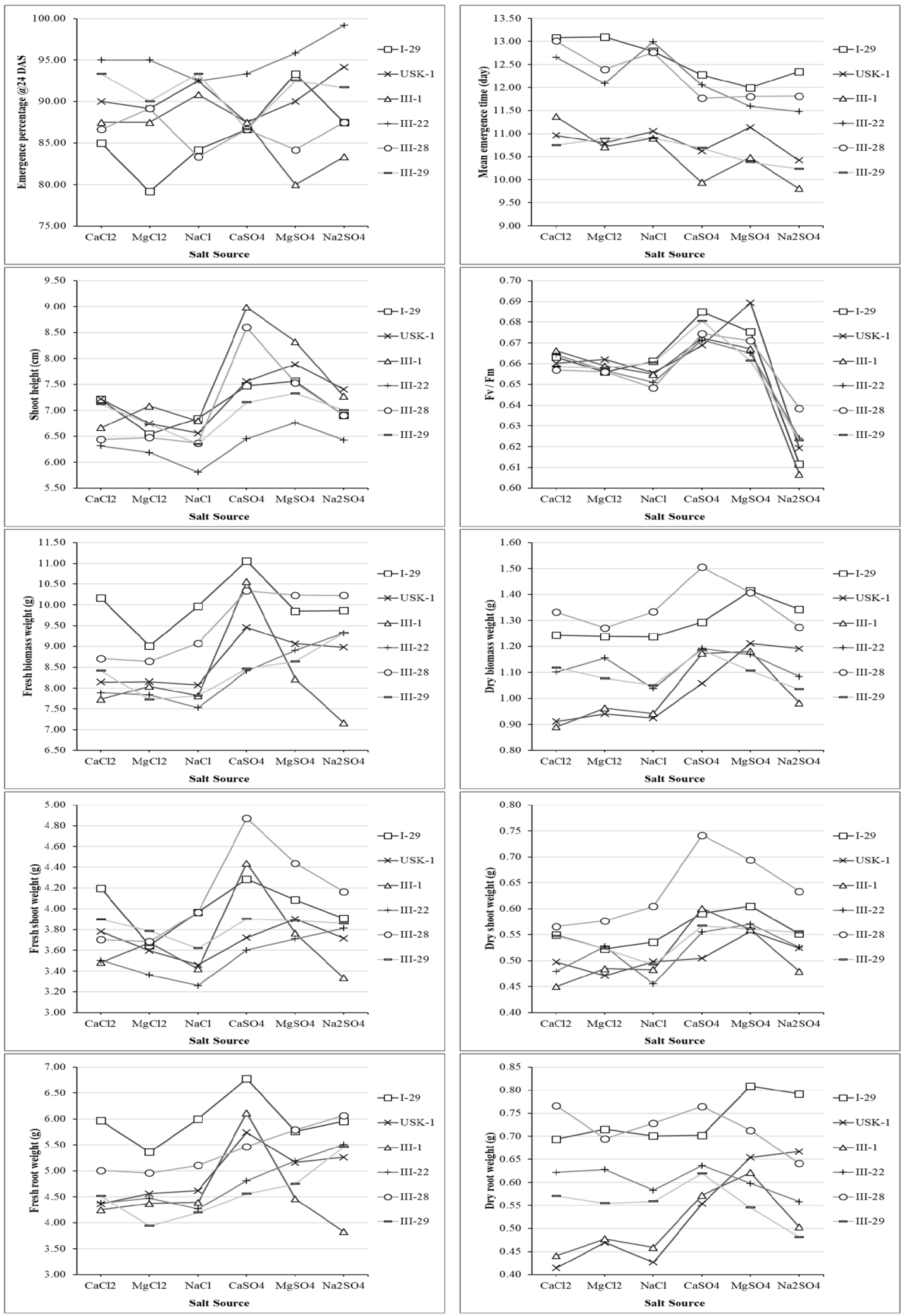

Figure 2. Comparison of faba bean genotypes under the main effect of salinity levels.

salt sources that most negatively affected the genotypes were $\mathrm{NaCl}$ and $\mathrm{Na}_{2} \mathrm{SO}_{4}$, it was determined that the salt source with less effect was determined for $\mathrm{CaSO}_{4}$ (Fig. 3). 


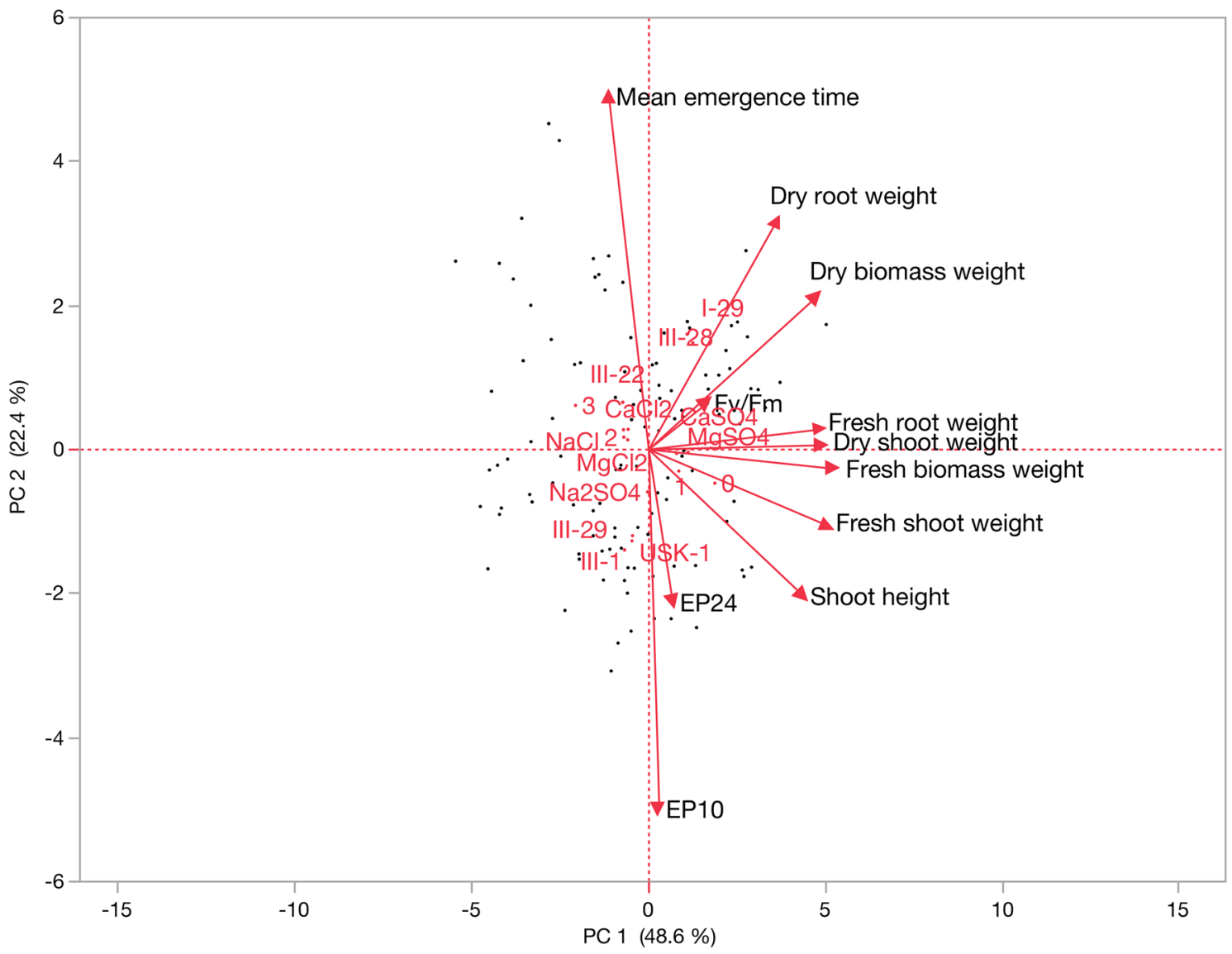

Figure 3. The principal component analysis (PCA) correlation of parameters with different salt sources and faba bean genotypes.

\begin{tabular}{|l|r|r|r|l|l|l|l|l|l|l|}
\hline & \multicolumn{1}{|c|}{ EP10 } & EP24 & MET & SH & FBW & DBW & FSW & DSW & FRW & DRW \\
\hline EP24 & $0.23^{* *}$ & & & & & & & & & \\
\hline MET & $-0.75^{* *}$ & $-0.19^{* *}$ & & & & & & & & \\
\hline SH & $0.37^{* *}$ & $0.17^{* *}$ & $-0.44^{* *}$ & & & & & & & \\
\hline FBW & $0.12^{* *}$ & $0.22^{* *}$ & $-0.24^{* *}$ & $0.72^{* *}$ & & & & & & \\
\hline DBW & $-0.15^{* *}$ & $0.09^{\text {ns }}$ & $-0.04^{\text {ns }}$ & $0.52^{* *}$ & $0.76^{* *}$ & & & & & \\
\hline FSW & $0.21^{* *}$ & $0.25^{* *}$ & $-0.34^{* *}$ & $0.80^{* *}$ & $0.91^{* *}$ & $0.68^{* *}$ & & & & \\
\hline DSW & $0.05^{\text {ns }}$ & $0.17^{* *}$ & $-0.24^{* *}$ & $0.59^{* *}$ & $0.77^{* *}$ & $0.82^{* *}$ & $0.80^{* *}$ & & & \\
\hline FRW & $0.06^{\text {ns }}$ & $0.19^{* *}$ & $-0.14^{* *}$ & $0.60^{* *}$ & $0.96^{* *}$ & $0.73^{* *}$ & $0.75^{* *}$ & $0.67^{* *}$ & & \\
\hline DRW & $-0.26^{* *}$ & $-0.01^{\text {ns }}$ & $0.13^{* *}$ & $0.33^{* *}$ & $0.55^{* *}$ & $0.89^{* *}$ & $0.42^{* *}$ & $0.47^{* *}$ & $0.59^{* *}$ & \\
\hline $\mathrm{F}_{\mathrm{v}} / \mathrm{F}_{\mathrm{m}}$ & $-0.03^{\text {ns }}$ & $-0.05^{\text {ns }}$ & $-0.01^{\text {ns }}$ & $0.20^{* *}$ & $0.19^{* *}$ & $0.21^{* *}$ & $0.23^{* *}$ & $0.20^{* *}$ & $0.15^{* *}$ & $0.16^{* *}$ \\
\hline
\end{tabular}

Table 8. Linear relationship based on correlation coefficient between investigated parameters. EP10 emergence percentage at 10th DAS, EP24 emergence percentage at 24th DAS, MET mean emergence time, SH shoot height, $F B W$ fresh biomass weight, $D B W$ dry biomass weight, FSW fresh shoot weight, $D S W$ dry shoot weight, $F R W$ fresh root weight, $D R W$ dry root weight. ${ }^{\star *}$ Significant at 0.01 probability level, $n s$ non-significant.

Relationship between investigated parameters. Statistical evaluation results ( $\mathrm{R}$ and $P$ values) of the linear relationships between investigated parameters are presented in Table 8 . There were significantly important $(P<0.01)$ positive correlations between parameters of shoot height, fresh biomass weight, dry biomass weight, fresh shoot weight, dry shoot weight, fresh root weight and dry root weight, although, most of the R values seems to be low which indicates a non-linear correlation. Mean emergence time has a negative correlation with all other investigated parameters except dry biomass weight and $\mathrm{F}_{\mathrm{v}} / \mathrm{F}_{\mathrm{m}}$ ratio (not significant), and dry root weight (a positive correlation). The substantial positive linear correlation was obtained between fresh biomass weight versus fresh root weight (96\%) and fresh shoot weight (91\%); dry biomass weight versus dry root weight (89\%) and dry shoot weight (82\%); shoot height versus fresh shoot weight $(80 \%)$ and fresh shoot weight versus dry shoot weight $(80 \%)$. The prominent negative relationship was observed between emergence percentage at 10 
DAS versus mean emergence time (-75\%). However, the relationship between emergence percentage at 10th DAS versus dry shoot weight, fresh root weight and $\mathrm{F}_{\mathrm{v}} / \mathrm{F}_{\mathrm{m}}$ ratio; emergence percentage at 24th DAS versus dry biomass weight, dry root weight and $\mathrm{F}_{\mathrm{v}} / \mathrm{F}_{\mathrm{m}}$ ratio; and mean emergence time versus dry biomass weight and $\mathrm{F}_{\mathrm{v}} /$ $\mathrm{F}_{\mathrm{m}}$ ratio was not statistically significant (Table 8).

\section{Discussion}

Final emergence percentages were always high (80-100\%) for III-22, III-29 and USK-1 faba bean genotypes. Mean emergence time values of I-29, III-1, III-29 and USK-1 faba bean genotypes did not show significant difference under $\mathrm{S} \times \mathrm{L}$ interaction, indicated that the emergence time of these faba bean genotypes was not affected from increasing salinity levels with all salt sources. However, seed emergence was significantly retarded; under high salinity level with $\mathrm{CaCl}_{2}$ and under medium and high salinity levels with $\mathrm{NaCl}$ salt source for both III-22 and III-28 faba bean genotypes. Similar results have been reported for common bean by Cavalcanti et al..$^{20}$ who studied the effect of water salinity (from 0.7 to $4.7 \mathrm{dS} / \mathrm{m}$ ), the ionic composition of $\mathrm{Na}+\mathrm{Mg}$, and their interaction. They did not observe a significant influence on the percent emergence and emergence speed index, which shows that different genotypes from the same species can have different behaviors.

In general, increasing salinity level regardless of the salt source resulted in decreases in shoot heights of these genotypes. Similarly, it was reported that the emergence and growth of castor bean were more affected by the salinity level than by the cationic composition of the irrigation water ${ }^{21}$. Also, it was stated that shoot height values of common bean genotypes were affected by salinity $(\mathrm{NaCl})$ only at $200 \mathrm{mM}^{13}$ whereas shoot height of pinto bean ${ }^{22}$ and emergence rate, shoot height and leaf area of castor bean ${ }^{23}$ were reduced by increased salinity $(\mathrm{NaCl})$ level. The latter researchers also concluded that the reduction in emergence, plant height, leaf area and chlorophyll $\mathrm{a}$ and $\mathrm{b}$ was much greater for an increase in salinity from 50 to $100 \mathrm{mM} \mathrm{NaCl}$ than that from 0 to $50 \mathrm{mM} \mathrm{NaCl}$.

Significant decreases on these parameters were observed especially under high salinity level with majority of the salt sources. The decreases were more pronuonced with $\mathrm{Cl}$ containing salt sources. Dry biomass weight values of III-1, III-22, III-29 and USK-1; dry shoot weight values of III-1, III-22 and USK-1; and dry root weight values of III-29 and USK-1 faba bean genotypes were significantly different under S $\times$ L interaction. The dry biomass and shoot weight values, in general, significantly decreased under high salinity level with especially some $\mathrm{Cl}$ containing and/or $\mathrm{Na}_{2} \mathrm{SO}_{4}$ salt sources whereas, no harmfull effect was observed at any salinity level with $\mathrm{CaSO}_{4}$ and $\mathrm{MgSO}_{4}$ salt sources. Del Pilar Cordovilla et al. ${ }^{24}$ reported that shoot and dry root weights of faba been at 25th days after onset of salt treatment were 0.76 and $0.35 \mathrm{~g}$ under control and $0.69 \mathrm{~g}$ under $100 \mathrm{mM}$ $\mathrm{NaCl}$ applications, repectively. Similarly, Helal and $\mathrm{Mengel}^{15}$ mentioned that $\mathrm{NaCl}$ salinity depresses faba been seedling growth and restricts protein formation, $\mathrm{CO}_{2}$ assimilation, and especially the incorporation of photosynthates into the lipid fraction.

It is explained that the photosynthetic performances of higher plants can vary widely according to growth forms, species, organs and stages concerning diverse eco-physiological demands $s^{25}$. Similarly, it was reported that exposure to salt did not have any immediate effect on $\mathrm{F}_{\mathrm{v}} / \mathrm{F}_{\mathrm{m}}$ in the Glycophyte Arabidopsis and the Halophyte Thellungiella species. However, during the development of salt stress over a $14 \mathrm{~d}$ period, this parameter fell in Arabidopsis exposed to either 100 or $150 \mathrm{~mm} \mathrm{NaCl}$. In Thellungiella, no change in $\mathrm{F}_{\mathrm{v}} / \mathrm{F}_{\mathrm{m}}$ occurred, even at the highest salt concentration ${ }^{26}$. In general, $\mathrm{F}_{\mathrm{v}} / \mathrm{F}_{\mathrm{m}}$ ratios under medium and high salinity levels with $\mathrm{Na}_{2} \mathrm{SO}_{4}$ salt source were the lowest almost for all faba bean genotypes.

Averaged over all salinity levels or salt sources, the performance order of faba bean genotypes was III- $28 \leq$ III$22 \leq$ I- $29<$ USK- $1 \leq$ III- $1 \leq$ III-29 on emergence percentage at 10 th DAS; I- $29 \leq$ III- $1 \leq$ III- $28<$ USK- $1 \leq$ III- $29<$ III22 on emergence percentage at 24th DAS; I-29 $<$ III- $28 \leq$ III- $22<$ USK-1 $\leq$ III- $29 \leq$ III- 1 on mean emergence time; III- $22<$ III- $29 \leq$ III- $28 \leq$ I- $29 \leq$ USK- $1<$ III- 1 on shoot height; III- $1 \leq$ III- $22 \leq$ III- $29 \leq$ USK- $1<$ III- $28<$ I- 29 on fresh biomass weight; III- $1 \leq$ USK- $1<$ III- $29 \leq$ III- $22<$ I- $29<$ III- 28 on dry biomass weight; III- $22<$ III- $1 \leq$ USK- $1<$ III$29<$ I- $29 \leq$ III- 28 on fresh shoot weight; USK- $1 \leq$ III- $1 \leq$ III- $22<$ III- $29 \leq$ I- $29<$ III- 28 on dry shoot weight; III$29 \leq$ III- $1<$ III- $22 \leq$ USK- $1<$ III- $28<$ I-29 on fresh root weight; III- $1 \leq$ USK- $1<$ III- $29<$ III- $22<$ III- $28 \leq$ I- 29 on dry root weight; and III- $1 \leq \mathrm{III}-22 \leq \mathrm{III}-29 \leq \mathrm{III}-28 \leq \mathrm{I}-29 \leq \mathrm{USK}-1$ on $\mathrm{F}_{\mathrm{v}} / \mathrm{F}_{\mathrm{m}}$ ratios. These performance orders clearly indicate that the response of cultivars or genotypes to salinity may differs for different growth parameters. Similarly, it was concluded that the growth responses of two faba cultivars (cv. Eresen 87, cv. Filiz 99) to two levels of $\mathrm{NaCl}$ salt was significantly different ${ }^{16}$. They revealed that Filiz 99 is slightly more $\mathrm{NaCl}$ resistant cultivar compared to $\mathrm{cv}$. Eresen 87. Large genetic variation of faba bean in tolerance to salinity has also been reported in different studies ${ }^{27,28}$.

Averaged over all faba bean genotypes, the order of salinity level in terms of negative effect was high $(3 \mathrm{dS} / \mathrm{m}) \geq \operatorname{medium}(2 \mathrm{dS} / \mathrm{m})>$ low $(1 \mathrm{dS} / \mathrm{m}) \geq \operatorname{control}(0.05 \mathrm{dS} / \mathrm{m})$ on emergence percentage at 10th and at 24 th DAS; high $(3 \mathrm{dS} / \mathrm{m})>$ medium $(2 \mathrm{dS} / \mathrm{m}) \geq$ low $(1 \mathrm{dS} / \mathrm{m}) \geq$ control $(0.05 \mathrm{dS} / \mathrm{m})$ on mean emergence time; high $(3 \mathrm{dS} / \mathrm{m})>$ medium $(2 \mathrm{dS} / \mathrm{m})>$ low $(1 \mathrm{dS} / \mathrm{m})>$ control $(0.05 \mathrm{dS} / \mathrm{m})$ on shoot height, fresh biomass weight, dry biomass weight and fresh root weight; high $(3 \mathrm{dS} / \mathrm{m})>$ medium $(2 \mathrm{dS} / \mathrm{m})>\operatorname{low}(1 \mathrm{dS} / \mathrm{m}) \geq \operatorname{control}(0.05 \mathrm{dS} / \mathrm{m})$ on fresh shoot weight and dry shoot weight; medium $(2 \mathrm{dS} / \mathrm{m}) \geq \operatorname{high}(3 \mathrm{dS} / \mathrm{m})>\operatorname{low}(1 \mathrm{dS} / \mathrm{m})>\operatorname{control}(0.05 \mathrm{dS} / \mathrm{m})$ on dry root weight; and high $(3 \mathrm{dS} / \mathrm{m})>$ medium $(2 \mathrm{dS} / \mathrm{m}) \geq \operatorname{low}(1 \mathrm{dS} / \mathrm{m}) \geq \operatorname{control}(0.05 \mathrm{dS} / \mathrm{m})$ on and $\mathrm{F}_{\mathrm{v}} / \mathrm{F}_{\mathrm{m}}$ ratio. Abd El-Baki and Mostafa ${ }^{17}$ observed that there was a sharp reduction in fresh and dry mass of shoots and roots of faba bean with increasing $\mathrm{NaCl}$ salinity. De Lima et al. ${ }^{21}$ and Nobre et al. ${ }^{29}$ observed that increasing water $\mathrm{NaCl}$ salinity (only with $\mathrm{NaCl}$ ) level caused significant decreases in percent emergence, emergence speed index and plant height values of caster bean. Similarly, for common bean, it was claimed that seedling growth at 200,250 and $300 \mathrm{mM} \mathrm{NaCl}$ were drastically affected with regard to control ${ }^{30}$. In general, growth reduction due to increased salinity illustrates the negative effect of salinity on plants, activated by altered osmotic potentials of salt in the root system that limits the gain of the required amount of water ${ }^{31,32}$. 
Similarly, averaged over all faba bean genotypes, the order of salt sources, in terms of negative effects, was $\mathrm{CaCl}_{2} \geq \mathrm{NaCl} \geq \mathrm{MgCl}_{2}>\mathrm{CaSO}_{4} \geq \mathrm{MgSO}_{4}>\mathrm{Na}_{2} \mathrm{SO}_{4}$ on emergence percentage at 10 th DAS; $\mathrm{CaSO}_{4} \geq \mathrm{MgCl}_{2} \geq \mathrm{MgSO}_{4} \geq \mathrm{NaCl} \geq \mathrm{CaCl}_{2} \geq \mathrm{Na}_{2} \mathrm{SO}_{4}$ on emergence percentage at 24th DAS; $\mathrm{CaCl}_{2} \geq \mathrm{NaCl} \geq \mathrm{MgCl}_{2}>\mathrm{MgSO}_{4} \geq \mathrm{CaSO}_{4} \geq \mathrm{Na}_{2} \mathrm{SO}_{4}$ on mean emergence time; $\mathrm{NaCl} \geq \mathrm{MgCl}_{2}>\mathrm{CaCl}_{2} \geq \mathrm{Na}_{2} \mathrm{SO}_{4}$ $>\mathrm{MgSO}_{4} \geq \mathrm{CaSO}_{4}$ on shoot height; $\mathrm{MgCl}_{2} \geq \mathrm{NaCl} \geq \mathrm{CaCl}_{2}>\mathrm{Na}_{2} \mathrm{SO}_{4} \geq \mathrm{MgSO}_{4}>\mathrm{CaSO}_{4}$ on fresh biomass weight; $\mathrm{NaCl} \geq \mathrm{CaCl}_{2} \geq \mathrm{MgCl}_{2}>\mathrm{Na}_{2} \mathrm{SO}_{4}>\mathrm{CaSO}_{4} \geq \mathrm{MgSO}_{4}$ on dry biomass weight; $\mathrm{NaCl} \geq \mathrm{MgCl}_{2} \geq \mathrm{CaCl}_{2} \geq \mathrm{Na}_{2} \mathrm{SO}_{4}>\mathrm{M}$ $\mathrm{gSO}_{4} \geq \mathrm{CaSO}_{4}$ on fresh shoot weight; $\mathrm{NaCl} \geq \mathrm{CaCl}_{2} \geq \mathrm{MgCl}_{2} \geq \mathrm{Na}_{2} \mathrm{SO}_{4}>\mathrm{MgSO}_{4} \geq \mathrm{CaSO}_{4}$ on dry shoot weight; $\mathrm{MgCl}_{2} \geq \mathrm{CaCl}_{2} \geq \mathrm{NaCl}>\mathrm{MgSO}_{4} \geq \mathrm{Na}_{2} \mathrm{SO}_{4}>\mathrm{CaSO}_{4}$ on fresh root weight; $\mathrm{NaCl} \geq \mathrm{CaCl}_{2} \geq \mathrm{MgCl}_{2} \geq \mathrm{Na}_{2} \mathrm{SO}_{4}>\mathrm{CaSO}_{4}$ $\geq \mathrm{MgSO}_{4}$ on dry root weight; and $\mathrm{Na}_{2} \mathrm{SO}_{4}>\mathrm{NaCl} \geq \mathrm{MgCl}_{2} \geq \mathrm{CaCl}_{2}>\mathrm{MgSO}_{4} \geq \mathrm{CaSO}_{4}$ on $\mathrm{F}_{\mathrm{v}} / \mathrm{F}_{\mathrm{m}}$ ratios. These orders show that, in general, the negative effects of $\mathrm{Cl}$ containing salt sources were higher than that of $\mathrm{SO}_{4}$ containing salt sources. In a study to investigate the effect of $\mathrm{Na}-, \mathrm{Cl}-$ and $\mathrm{NaCl}$-treated soil on faba bean, it was concluded that both high $\mathrm{Na}$ and high $\mathrm{Cl}$ reduced growth of faba bean but plants were more sensitive to $\mathrm{Cl}$ than to $\mathrm{Na}^{33}$. The researchers also mentioned that reductions in growth and photosynthesis were greater under $\mathrm{NaCl}$ stress and the effect was mainly additive. Also, they claim that salinity caused by high concentrations of $\mathrm{NaCl}$ can reduce growth by the accumulation of high concentrations of both $\mathrm{Na}$ and $\mathrm{Cl}$ simultaneously, but the effects of the two ions may differ. It was reported that the emergence speed index and the emergence percentage of castor bean seedlings were significantly affected by the studied types of water salinity ${ }^{21}$. They claimed that the order of the cations in the irrigation water, in terms of negative effects, was $\mathrm{Na}>\mathrm{Na}+\mathrm{Ca}>\mathrm{Ca}>\mathrm{Na}+\mathrm{Ca}+\mathrm{Mg}>\mathrm{K}$. In another study, it was concluded that common bean seedling growth decreases in different type of salinity, due to the seedlings being unable to adjust osmotically or due to the toxic effects of $\mathrm{Cl}, \mathrm{SO}_{4}$, and/or $\mathrm{Na}^{34}$. They observed that inhibiting effects of $\mathrm{Na}_{2} \mathrm{SO}_{4}$ on shoot height, fresh biomass and root weight parameters were by $20 \%$ stronger than those of $\mathrm{NaCl}$. However, in our experiment negative effects of $\mathrm{NaCl}$ on these parameters were somewhat higher than those of $\mathrm{Na}_{2} \mathrm{SO}_{4}$ salt source.

Principal Component Analysis was performed to examine how the genotypes differ in terms of salt sources and salinity levels. It was determined that III-28 and I-29 genotypes were more tolerant to $\mathrm{MgSO}_{4}$ and CaSO 4 salt sources and were better explained by dry root weight, dry biomass weight and $\mathrm{F}_{\mathrm{v}} / \mathrm{F}_{\mathrm{m}}$ parameters. The III-22 genotype had a late mean emergence time than the others. III-29, III-1 and USK-1 genotypes have more sensitivity to salinity than I-29 and III-28 genotypes with all salt sources. In studies, investigating salinity stress in faba bean, principal component analysis was carried out in limited numbers. However, there are a few studies evaluating common parameters with this study. Rajhi et al. ${ }^{28}(2020)$ tested salinity $(\mathrm{NaCl})$ resistance in six different bean genotypes. It was reported that mass of the fresh root, mass of fresh shoot, mass of total fresh plant, mass of the dry root, length of the root and length of the shoot parameters explained the phenotypic variance according to $\mathrm{PCA}^{28}$. In another study using $\mathrm{NaCl}$ salt source in faba bean, it was reported that the parameters evaluated by PCA, root dry weight, shoot fresh weight, shoot dry weight, root fresh weight, dry seed weight and fresh seed weight were collected in one group ${ }^{35}$. The distribution of parameters is similar to our study.

Salinity is an ever-present major constraint and a major threat to most crops, particularly in areas with irrigated agriculture. It is quite evident that salt stress also significantly affects legume crops. Suitability of poor quality water as a supplemental source for irrigation depends on the level of salinity and solute concentration in the water and the selected crop. Even genotypes of the same species may demonstrate distinct responses to the amount and kind of salts present in irrigation and/or soil, especially during the emergence and early seedling growth. There is an immense need about the knowledge of genotype response to both salt source and salinity level for higher yield across saline environments. This study showed that faba bean genotypes have different behaviors in terms of response to the increasing salinity levels artificially makeup by using different salt sources indicating that salt response of faba bean is genotype-specific. These results provide support for further studies on the effect of the salt sources with different salinity level on emergence and early seedling growth, but these still need to be tied into a general response of the genotypes during vegetative and reproductive phases under these conditions.

\section{Methods}

Experimental site and plant material. The experiment was carried out in plastic seed trays at Akdeniz University Experimental Research Area of Agricultural Faculty, Antalya, located in the south-west region of Turkey, latitude of $36^{\circ} 53^{\prime} 15^{\prime \prime}$ North and longitude of $30^{\circ} 38^{\prime} 53^{\prime \prime}$ East and $58 \mathrm{~m}$ above the sea level. Seeds of six faba bean genotypes including I-29, III-1, III-22, III-28, III-29 and USK-1 which are widely extended varieties in Turkey were used in this study. Some traits of plant materials were given in Table 9.

Plastic trays, of dimension $50 \times 30 \times 5 \mathrm{~cm}$ (length $\times$ width $\times$ height) each containing 45 cells $(4.5 \mathrm{~cm}$ deep, 5.0 and $4.0 \mathrm{~cm}$ upper and bottom diameter per cell), were used for seed emergence and seedling development. Soils used in the experiment as a sowing substrate were sieved with a $4 \mathrm{~mm}$ screen in order to remove large particles and filled to the cells of each seed tray. The experimental soil used in the experiment had sandy-loam with $54.8 \%$ sand, $16.9 \%$ silt and $28.3 \%$ clay particles with a bulk density of $1.43 \mathrm{~g} / \mathrm{cm}^{3}$. The original electrical conductivity and $\mathrm{pH}$ of the saturated soil paste extract were $0.37 \mathrm{dS} / \mathrm{m}$ and 7.63 , respectively. Soil water contents at field capacity and permanent wilting point were measured as 23.8 and $9.4 \%$, respectively.

Experimental design and treatments. The experimental factors were six salt sources $\left(\mathrm{CaCl}_{2}, \mathrm{MgCl}_{2}\right.$, $\mathrm{NaCl}, \mathrm{CaSO}_{4}, \mathrm{MgSO}_{4}$, and $\left.\mathrm{Na}_{2} \mathrm{SO}_{4}\right)$, three irrigation water salinity levels with an electrical conductivity $\left(\mathrm{EC}_{\mathrm{w}}\right)$ of 1.0 (low), 2.0 (medium) and $3.0 \mathrm{dS} / \mathrm{m}$ (high) in addition to distilled water with an electrical conductivity of $0.05 \mathrm{dS} / \mathrm{m}$ without any salt source as a control and six faba bean genotypes (I-29, III-29, III-1, III-22, III-28 and USK-1).

At the beginning of the experiment, the amount of salt required for each salt source to generate the desired electrical conductivity values in irrigation waters was determined in the laboratory. Considering the determined 


\begin{tabular}{|l|l|l|l|l|}
\hline Genotypes & Origin & Plant height $(\mathbf{c m})$ & Seed yield $(\mathbf{k g} / \mathbf{d a})$ & 100-Seed weight $(\mathbf{g r})$ \\
\hline I-29 & ICARDA ${ }^{*}$ & 45.8 & 45.0 & 94.1 \\
\hline III-1 & ICARDA & 42.8 & 75.0 & 106.0 \\
\hline III-22 & ICARDA & 45.0 & 50.0 & 88.4 \\
\hline III-28 & ICARDA & 38.5 & 95.0 & 93.5 \\
\hline III-29 & ICARDA & 50.3 & 75.0 & 80.4 \\
\hline USK-1 & ICARDA & $*$ & $*$ & $*$ \\
\hline
\end{tabular}

Table 9. Some morphological traits of genotypes. ${ }^{* \star}$ International Center for Agricultural Research in the dry areas, ${ }^{*}$ not evaluated.

amounts, irrigation waters with targeted three different salinity levels for each salt source were made separately ready for use in the experimental area by using 18 plastic water tanks having 100-l volume capacity.

There were three replications with 30 seeds for each salt source and salinity level combinations of each faba bean genotype. Therefore, a total of 570 ([ 3 salinity levels $\times 6$ salt sources +1 control] $\times 3$ replication $\times 30$ seed per replication) seeds were used for each faba bean genotype. On the day of sowing, the seeds of uniform size were selected and sown in growth substrate with one seed per cell at a depth of $10 \mathrm{~mm}$ depth. Starting from the sowing, each seed tray was equally watered every day considering the salt source and salinity level treatment combination.

Analyses and measurements. Starting on the 9th day after sowing (DAS) until the experiment lasted at 24th DAS, the number of emerged seeds at each replication was recorded every 24 -h. Emergence percentage (EP) was calculated at daily periods as the percent ratio of the number of emerged seeds to the number of total seeds for each replication. Similarly, mean emergence time (MET) was calculated at the end of the experiment as the time (day) average value of all emerged seeds for each replication ${ }^{36}$.

Before terminating the experiment, four replicate fluorescence measurements for each seedling were realized in the middle part of intact undetached leaf blades by using a fluorometer (PAR-FluorPen FP 110/D). The potential quantum efficiency of photosystem II (PSII) was determined using expressions $\left.\mathrm{F}_{\mathrm{v}} / \mathrm{F}_{\mathrm{m}}=\left(\mathrm{F}_{\mathrm{m}}-\mathrm{F}_{0}\right) / \mathrm{F}_{\mathrm{m}}\right)$, in which $\mathrm{F}_{\mathrm{v}}, \mathrm{F}_{0}$ and $\mathrm{F}_{\mathrm{m}}$ are variable, minimal and maximal levels of chlorophyll fluorescence. This is a relative measurement of the PSII, and can, therefore, be used to measure the performance of photosynthesis within a leaf where an $\mathrm{F}_{\mathrm{v}} / \mathrm{F}_{\mathrm{m}}$ value near to 0.8 is regarded as healthy in most plants ${ }^{37}$. Reduction in the $\mathrm{F}_{\mathrm{v}} / \mathrm{F}_{\mathrm{m}}$ ratio indicates that the plant is subjected to biotic and/or abiotic stresses ${ }^{38}$. After these measurements, all seedlings were removed from seed tray holes and cleaned with water to remove soils from their roots. The shoots and roots of seedlings were separated by cutting tissues with a razor blade on connecting lines that could be easily seen with a different color of the stem and root tissues. Then, the distance between the cutting point and tip of the longest leaves was measured as shoot heights and fresh and oven-dried (at $70^{\circ} \mathrm{C}$ until a constant value) shoot and root weight of the seedlings were obtained.

Statement on guidelines. All experimental procedures and field studies on plants/seeds comply with relevant institutional, national, and international guidelines and legislation.

Data analysis. Data on the emergence, seedling growth variables and $\mathrm{F}_{\mathrm{v}} / \mathrm{F}_{\mathrm{m}}$ ratio were statistically analyzed using Analysis of Variance (ANOVA) with SPSS 13.0 (SPSS Inc., Chicago, IL). The main effects of the interactions between salt source and irrigation water salinity level on investigated parameters were analyzed by univariate regression using SPSS 13.0. Homogeneity and normality were checked before subjecting data to ANOVA. Unless otherwise noted, all statistical tests were performed at the 0.01 level of significance. If ANOVA reported significant differences, treatment mean differences were separated using by Duncan's multiple range tests at $P \leq$ 0.05. Principal Component Analysis was performed on JMP Pro 16.0.0 (SAS Institute Inc.). Considering correlation coefficient $(\mathrm{R})$ values, the strengths of the linear relationships between investigated parameters were evaluated as strong $(\mathrm{R} \geq 0.8)$, moderate $(0.5<\mathrm{R}<0.8)$ and weak $(\mathrm{R} \leq 0.5)^{39}$.

Received: 28 December 2020; Accepted: 31 August 2021

Published online: 14 September 2021

\section{References}

1. Duc, G. Faba bean (Vicia faba L.). Field Crops Res. 53, 99-109 (1997).

2. Łabuda, H. Flowering and characteristics of useful traits of some faba bean (Vicia faba L. var. major Harz) cultivars and breeding lines. Acta Agrobot. 65, 139-148 (2012).

3. Haciseferoğullari, H., Gezer, I., Bahtiyarca, Y. \& Mengeş, H. O. Determination of some chemical and physical properties of Sakiz faba bean (Vicia faba L. Var. major). J. Food Eng. 60, 475-479 (2003).

4. FAOSTAT. Crop Statistics (2020). http://faostat.fao.org.

5. Pekşen, A., Pekşsen, E. \& Artık, C. Bazı bakla (Vicia faba L.) populasyonlarının bitkisel özellikleri ve taze bakla verimlerinin belirlenmesi (Determination of plant characteristics and green pod yield of some faba bean (Vicia faba L.) populations). Anadolu Tarim Bilim. Derg. 21, 225-230 (2006).

6. TUIK. Crop Production Statistics (Vegetables) (Turkish Statistical Institute, 2020). https://biruni.tuik.gov.tr $/ \mathrm{medas} /$ ?kn=92\&locale= tr. 
7. Hanson, B. R., Grattan, S. R. \& Fulton, A. Agricultural Salinity and Drainage. Division of Agriculture and Natural Resources Publication 3375 (Department of Land, Air and Water Resources, University of California, 2006). https://doi.org/10.1016/S0016-0032(73) 90636-4.

8. Kurunc, A. et al. Effects of salt source and irrigation water salinity on growth, yield and quality parameters of Stevia rebaudiana Bertoni. Sci. Hortic. (Amst.) 270, 1-10 (2020).

9. Fernández-García, N., Martínez, V. \& Carvajal, M. Effect of salinity on growth, mineral composition, and water relations of grafted tomato plants. J. Plant Nutr. Soil Sci. 167, 616-622 (2004).

10. Dişli, Y. Antalya İli Kale (Demre) ilçesi yer altı sulama suyu kalitesi üzerine bir araștırma (A Research on the Subsurface Irrigation Water Quality Of Kale (Demre) District of Antalya Province) (Selçuk Üniversitesi, 1997).

11. Maas, E. V. \& Hoffman, G. J. Crop salt tolerance-Current assessment. J. Irrig. Drain. Div. 103, 115-134 (1977).

12. Ayers, R. S. \& Westcot, D. W. Water Quality for Agriculture-FAO Irrigation and Drainage Paper 29 (FAO_Food and Agriculture Organization of the United Nations, 1985).

13. Kouam, E. B., Ndo, S. M., Mandou, M. S., Chotangui, A. H. \& Tankou, C. M. Genotypic variation in tolerance to salinity of common beans cultivated in Western Cameroon as assessed at germination and during early seedling growth. Open Agric. 2, 600-610 (2017).

14. Nadeem, M. et al. Grain legumes and fear of salt stress: Focus on mechanisms and management strategies. Int. J. Mol. Sci. 20, 1-27 (2019).

15. Helal, H. M. \& Mengel, K. Interaction between light intensity and $\mathrm{NaCl}$ salinity and their effects on growth, $\mathrm{CO}_{2}$ assimilation, and photosynthate conversion in young broad beans. Plant Physiol. 67, 999-1002 (1981).

16. Bulut, F. \& Akinci, S.. The effect of salinity on growth and nutrient composition in broad bean (Vicia faba L.) seedlings. Fresenius Environ. Bull. 19, 2901-2910 (2010).

17. Abd El-Baki, G. K. \& Mostafa, D. The potentiality of Trichoderma harzianum in alleviation the adverse effects of salinity in faba bean plants. Acta Biol. Hung. 65, 451-468 (2014).

18. Hasanuzzaman, M., Nahar, K. \& Fujita, M. Plant response to salt stress and role of exogenous protectants to mitigate salt-induced damages BT-Ecophysiology and responses of plants under salt stress. In Ecophysiology and Responses of Plants under Salt Stress (eds Ahmad, P. et al.) 25-87 (Springer, 2013). https://doi.org/10.1007/978-1-4614-4747-4_2.

19. Cohen, B. H. Explaining Psychological Statistics (Wiley, 2008).

20. Cavalcanti, M. L. F. et al. Tolerância da mamoneira BRS 149 à salinidade: Germinação e características de crescimento. Rev. Bras. Eng. Agríc. Ambient. 9, 57-61 (2005).

21. De Lima, G. S. et al. Emergence, growth, and flowering of castor beans as a function of the cationic composition of irrigation water. Semin. Agrar. 37, 651-664 (2016).

22. Gholami, A., Sharafi, S., Sharafi, A. \& Ghasemi, S. Germination of different seed size of pinto bean cultivars as affected by salinity and drought stress. J. Food Agric. Environ. 7, 555-558 (2009).

23. Guo, X., Zhou, G., Zhu, G. \& Jiao, X. Effects of calcium on emergence and seedling growth of castor bean under salinity stress. Curr. Sci. 116, 2028-2035 (2019).

24. Del Pilar Cordovilla, M., Ligero, F. \& Lluch, C. Effects of $\mathrm{NaCl}$ on growth and nitrogen fixation and assimilation of inoculated and $\mathrm{KNO}_{3}$ fertilized Vicia faba L. and Pisum sativum L. plants. Plant Sci. 140, 127-136 (1999).

25. Osmond, C. B., Winter, K. \& Ziegler, H. Functional significance of different pathways of $\mathrm{CO}_{2}$ fixation in photosynthesis BT-Physiological plant ecology II: Water relations and carbon assimilation. In Physiological Plant Ecology II (eds Lange, O. L. et al.) 479-547 (Springer, 1982). https://doi.org/10.1007/978-3-642-68150-9_16.

26. Stepien, P. \& Johnson, G. N. Contrasting responses of photosynthesis to salt stress in the glycophyte arabidopsis and the halophyte thellungiella: Role of the plastid terminal oxidase as an alternative electron sink. Plant Physiol. 149, 1154-1165 (2009).

27. Tavakkoli, E., Paull, J., Rengasamy, P. \& McDonald, G. K. Comparing genotypic variation in faba bean (Vicia faba L.) in response to salinity in hydroponic and field experiments. Field Crops Res. 127, 99-108 (2012).

28. Rajhi, I. et al. Photosynthetic and physiological responses of small seeded faba bean genotypes (Vicia faba L.) to salinity stress: Identification of a contrasting pair towards salinity. Photosynthetica 58, 174-185 (2020).

29. Nobre, R. G., de Lima, G. S., Gheyi, H. R., da Lourenço, G. S. \& dos Soares, L. A. A. Emergência, crescimento e produção da mamoneira sob estresse salino e adubação nitrogenada. Rev. Cienc. Agron. 44, 76-85 (2013).

30. Mena, E. et al. Effect of salt stress on seed germination and seedlings growth of Phaseolus vulgaris L. Cultiv. Trop. 36, 71-74 (2015).

31. Mer, R. K., Prajith, P. K., Pandya, D. H. \& Pandey, A. N. Effect of salts on germination of seeds and growth of young plants of Hordeum vulgare, Triticum aestivum, Cicer arietinum and Brassica juncea. J. Agron. Crop Sci. 185, 209-217 (2000).

32. Rhoades, J. D., Kandiah, A. \& Mashali, A. M. The Use of Saline Waters for Crop Production. FAO Irrigation and Drainage Paper 48 (Food and Agriculture Organization of The United Nations, 1992).

33. Tavakkoli, E., Rengasamy, P. \& Mcdonald, G. K. High concentrations of $\mathrm{Na}^{+}$and $\mathrm{Cl}^{-}$ions in soil solution have simultaneous detrimental effects on growth of faba bean under salinity stress. J. Exp. Bot. 61, 4449-4459 (2010).

34. Kaymakanova, M. Effect of salinity on germination and seed physiology in bean (Phaseolus vulgaris L.). Biotechnol. Biotechnol. Equip. 23, 326-329 (2009).

35. Latef, A. A. A., Hasanuzzaman, M. \& Tahjib-Ul-Arif, M. Mitigation of salinity stress by exogenous application of cytokinin in faba bean (Vicia faba L.). Not. Bot. Horti Agrobot. Cluj-Napoca 49, 12192 (2021).

36. Ellis, R. H. \& Roberts, E. H. The quantification of ageing and survival in orthodox seeds. Seed Sci. Technol. 9, 373-409 (1981)

37. Maxwell, K. \& Johnson, G. N. Chlorophyll fluorescence-A practical guide. J. Exp. Bot. 51, 659-668 (2000).

38. Baker, N. R. Chlorophyll fluorescence: A probe of photosynthesis in vivo. Annu. Rev. Plant Biol. 59, 89-113 (2008).

39. Peck, R. \& Devore, J. Statistics: The Exploration \& Analysis of Data (Brooks/Cole, Cengage Learning, 2012).

\section{Acknowledgements}

The authors would like to acknowledge the ICARDA (International Center for Agricultural Research in the Dry Areas) for providing seeds of faba bean genotypes.

\section{Author contributions}

N.B. and A.K. conceived and designed the experiments; H.S. provided the study materials; A.K., N.B., K.H.D. and M.A. performed the experiments and collected the data; A.K. and H.S. analyzed the data and interpreted the results; H.S., A.K. and N.B. wrote the paper.

\section{Competing interests}

The authors declare no competing interests.

\section{Additional information}

Correspondence and requests for materials should be addressed to A.K. 
Reprints and permissions information is available at www.nature.com/reprints.

Publisher's note Springer Nature remains neutral with regard to jurisdictional claims in published maps and institutional affiliations.

(c) (i) Open Access This article is licensed under a Creative Commons Attribution 4.0 International License, which permits use, sharing, adaptation, distribution and reproduction in any medium or format, as long as you give appropriate credit to the original author(s) and the source, provide a link to the Creative Commons licence, and indicate if changes were made. The images or other third party material in this article are included in the article's Creative Commons licence, unless indicated otherwise in a credit line to the material. If material is not included in the article's Creative Commons licence and your intended use is not permitted by statutory regulation or exceeds the permitted use, you will need to obtain permission directly from the copyright holder. To view a copy of this licence, visit http://creativecommons.org/licenses/by/4.0/.

(C) The Author(s) 2021 